\title{
Singularités quasi-ordinaires toriques et polyèdre de Newton du discriminant
}

\author{
P. D. González Pérez
}

\begin{abstract}
Nous étudions les polynômes $F \in \mathbb{C}\left\{S_{\tau}\right\}[Y]$ à coefficients dans l'anneau de germes de fonctions holomorphes au point spécial d'une variété torique affine. Nous généralisons à ce cas la paramétrisation classique des singularités quasi-ordinaires. Cela fait intervenir d'une part une généralization de l'algorithme de Newton-Puiseux, et d'autre part une relation entre le polyèdre de Newton du discriminant de $F$ par rapport à $Y$ et celui de $F$ au moyen du polytope-fibre de Billera et Sturmfels [3]. Cela nous permet enfin de calculer, sous des hypothèses de non dégénérescence, les sommets du polyèdre de Newton du discriminant a partir de celui de $F$, et les coefficients correspondants à partir des coefficients des exposants de $F$ qui sont dans les arêtes de son polyèdre de Newton.
\end{abstract}

\section{Introduction}

Le sujet de la première partie de ce travail est la représentation des racines $Y(X)$ d'une équation polynôme $F\left(X_{1}, \ldots, X_{d} ; Y\right)=0$ par des séries à exposants fractionnaires en les variables $X=\left(X_{1}, \ldots, X_{d}\right)$. Il s'agit de généraliser le théorème de Newton-Puiseux. Nous poursuivrons dans une direction inaugurée par McDonald dans [9], et précisons ses résultats.

Notre approche est d'étudier d'abord le problème dans le cas d'un polynôme $F \in$ $\mathbb{C}\left\{S_{\tau}\right\}[Y]$, où $\mathbb{C}\left\{S_{\tau}\right\}$ est l'anneau des germes des fonctions holomorphes au point spécial d'une variété torique affine correspondant à un cône rationnel strictement convexe, $\tau \subset$ $\left(\mathbb{R}^{d}\right)^{*}$, de dimension $d$. Nous résolvons le probleme, lorsque le discriminant $\Delta_{Y} F$ de $F$ par rapport à $Y$ est de la forme $X^{u} \epsilon$ où $\epsilon$ est une unité dans $\mathbb{C}\left\{S_{\tau}\right\}$ et $u$ appartient au semigroupe $S_{\tau}:=\tau^{\vee} \cap \mathbb{Z}^{d}$ des élements de $\mathbb{Z}^{d}$ qui appartiennent au cône dual $\tau^{\vee}:=\left\{w \in \mathbb{R}^{d} /\langle w, u\rangle \geq\right.$ $0, \forall u \in \tau\}$. Ceci est en fait une généralisation de l'étude classique des singularités quasiordinaires, qui correspondent au cas où $\tau$ est le quadrant positif.

La réduction du cas général à ce cas fait appel à des constructions combinatoires sur le polyèdre de Newton $\mathcal{N}(F) \subset \mathbb{R}^{d+1}$ de $F$. La plus importante, déjà utilisée dans [9] est celle du polyèdre-fibre $Q(F) \subset \mathbb{R}^{d}$ de $\mathcal{N}(F)$ par rapport à sa projection $\mathcal{N}(F) \rightarrow \mathbb{R}^{d}$ sur l'espace des exposants des monômes en $X$. Les points extrêmes de $Q(F)$ correspondent à certains chemins dans les arêtes de $\mathcal{N}(F)$.

Le polyèdre-fibre est également relié au polyèdre de Newton de $F$ du discriminant de $F$ par rapport à $Y$. Si $F=a_{0}(X)+\cdots+a_{r}(X) Y^{r}$, on a l'inclusion de polyèdres de Newton

$$
\mathcal{N}\left(\Delta_{Y} F\right)+\mathcal{N}\left(a_{0}\right)+\mathcal{N}\left(a_{r}\right) \subseteq \mathcal{Q}(F)
$$

(où la somme est la somme de Minkowski), avec l'égalité sous des hypothèses de nondégénérescence des coefficients de $F$ par rapport à $\mathcal{N}(F)$, (théorème 4). 
Un cône $\tau \subset\left(\mathbb{R}^{d}\right)^{*}$ est compatible avec des polyèdres $\mathcal{P}_{1}, \ldots, \mathcal{P}_{s} \subset \mathbb{R}^{d}$ s'il est constitué des fonctions linéaires qui prennent toutes leur valeur minimale sur $\mathcal{P}_{1}, \ldots, \mathcal{P}_{s}$ en des point fixés $p_{1} \in \mathcal{P}_{1}, \ldots, p_{s} \in \mathcal{P}_{s}$. On décrit le résultat principal, le théorème 3 , dans le cas où $F$ est un polynôme réduit dans l'anneau $\mathbb{C}\{X\}[Y]$. Si $\tau \subset\left(\mathbb{R}_{+}^{d}\right)^{\vee}$ est un cône rationnel strictement convexe de dimension $d$ compatible avec les polyèdres $\mathcal{N}\left(\Delta_{Y} F\right), \mathcal{N}\left(a_{r}\right)$ et $\mathcal{Q}(F)$, alors l'homomorphisme $\mathbb{C}\{X\} \rightarrow \mathbb{C}\left\{S_{\tau}\right\}$ étendant l'inclusion des algèbres $\mathbb{C}\left[\mathbb{Z}_{+}^{d}\right] \rightarrow \mathbb{C}\left[S_{\tau}\right]$ transforme $F$ en un polynôme $F_{\tau} \in \mathbb{C}\left\{S_{\tau}\right\}[Y]$ dont toutes les racines sont de la forme $X^{u} \varepsilon(X)$ où $u \in \frac{1}{k} Z^{d}, \varepsilon(X)$ est une unité dans l'anneau $\mathbb{C}\left\{\frac{1}{k} S_{\tau}\right\}$ et $k$ est un entier positif. La construction des racines est donnée par un algorithme qui généralise celui du théorème de Newton-Puiseux (théorème 2), et qui pourrait se développer à l'aide d'un logiciel de calcul formel. On peut comparer l'algorithme obtenu avec celui de [2], developpé pour le cas quasi-ordinaire.

Nous utilisons ces résultats pour donner une description des sommets du polyèdre de Newton du résultant $\operatorname{Res}_{Y}(F, G)$ des polynômes $F, G \in \mathbb{C}\left\{S_{\tau}\right\}$ à partir des polyèdres-fibres $\mathcal{Q}(F), Q(G)$ et $Q(F G)$; nous calculons enfin les coefficients des monômes correspondants aux sommets du polyédre de Newton de $a_{0} a_{r} \Delta_{Y} F$ et de $\operatorname{Res}_{Y}(F, G)$ sous des hypothèses de non dégénérescence. Ces coefficients dépendent entre autres des résultants des paires de polynômes à une variable obtenus en-regardant de $F$ et de $G$ que les termes donts les exposants appartiennent à des paires parallèles d'arêtes de $\mathcal{N}(F)$ et $\mathcal{N}(G)$. On montre un résultat analogue pour le discriminant. Nous trouvons aussi, avec une méthode très différente, des résultats de même nature que ceux de Gel'fand, Kapranov et Zelevinski dans [6].

\section{Paramétrisation de singularités quasi-ordinaires toriques}

\subsection{L'algèbre des germes de fonctions holomorphes au point distingué d'une variété to- rique affine}

Soit $\tau$ un cône convexe rationnel de dimension $d$ dans $\left(\mathbb{R}^{d}\right)^{*}$. Cette condition garantit que le cône dual $\tau^{\vee}:=\left\{w \in \mathbb{R}^{d} /\langle w, u\rangle \geq 0, \forall u \in \tau\right\}$ est un cône rationnel strictement convexe. Pour cette raison, chaque élément de $S_{\tau}$ peut s'exprimer commme somme d'éléments du semigroupe $S_{\tau}$ d'un nombre fini de manières. L'ensemble des séries formelles à exposants dans $S_{\tau}$ est un anneau, que nous notons par $\mathbb{C}\left[\left[S_{\tau}\right]\right]$. La propriété de finitude précédente permet de garantir que les coefficients de la série produit sont des fonctions polynomiales des coefficients des facteurs. Ces anneaux sont définis dans [9], pour construire des racines d'un polynôme $F \in \mathbb{C}\left[X_{1}, \ldots, X_{d}\right][Y]$, à la Newton-Puiseux.

On va donner une interprétation géométrique de ces anneaux au moyen de la variété torique associée au cône $\tau$ dans le cas où $\tau$ est un cône rationnel strictement convexe de dimension $d$ dans $\mathbb{R}^{d}$.

Soit $N \subset\left(\mathbb{R}^{d}\right)^{*}$ un réseau de dimension $d$, de réseau dual $M \subset \mathbb{R}^{d}$. Le cône $\tau$ définit le semi-groupe de type fini $S_{\tau}:=\tau^{\vee} \cap M$. Soit $\mathbb{C}\left[S_{\tau}\right]$ l'algèbre du semi-groupe $S_{\tau}$ à coefficients dans $\mathbb{C}$. Associons à $(\tau, N)$ la variété torique affine $Z_{\tau}:=\operatorname{Spec} \mathbb{C}\left[S_{\tau}\right]$. Chaque point fermé de $Z_{\tau}$ est définit par un homomorphisme de semi-groupes $S_{\tau} \rightarrow \mathbb{C}$. La valeur de la fonction $X^{u} \in \mathbb{C}\left[S_{\tau}\right]$ au point $x$ est $x(u)$. L'orbite de dimension 0 de la variété $Z_{\tau}$ est le point spécial $z_{\tau}$ défini par l'homomorphisme de semi-groupes $S_{\tau} \rightarrow \mathbb{C}$ qui applique $0 \mapsto 1$ et $u \mapsto 0$ si $u \neq 0$ (pour tout ceci, voir [4] ou [10]).

L'anneau des séries convergentes à exposants dans $S_{\tau}$, que nous notons par $\mathbb{C}\left\{S_{\tau}\right\}$, est 
l'ensemble des séries de $\mathbb{C}\left[\left[S_{\tau}\right]\right]$ qui sont absolument convergentes dans un voisinage du point spécial $z_{\tau}$ de la variété torique $Z_{\tau}$. Si $\tau$ est un cône convexe rationnel de dimension $d$, on défini $\mathbb{C}\left\{S_{\tau}\right\}=\bigcap \mathbb{C}\left\{S_{\sigma}\right\}$ où $\sigma$ parcourt les cônes rationnels strictement convexes de dimension $d$ contenus dans $\tau$.

Lemme 1 L'algèbre locale des germes de fonctions holomorphes au point $z_{\tau}$ de $Z_{\tau}$ est isomorphe à $\mathbb{C}\left\{S_{\tau}\right\}$.

Preuve Soient $u_{1}, \ldots, u_{s}$ des générateurs du semi-groupe $S_{\tau}$. L'homomorphisme $\mathbb{C}\left[U_{1}, \ldots, U_{s}\right] \rightarrow \mathbb{C}\left[S_{\tau}\right]$, défini par $U_{i} \mapsto X^{u_{i}} \in \mathbb{C}\left[S_{\tau}\right]$ est surjectif. Son noyau est un idéal premier $I$. Ce morphisme définit un plongement $Z_{\tau} \subset \mathbb{C}^{s}$ de la variété torique affine $Z_{\tau}:=\operatorname{Spec} \mathbb{C}\left[S_{\tau}\right]$ définie par le cône $\tau$, et l'image du point distingué $z_{\tau}$ est l'origine de $\mathbb{C}^{s}$. Soit $R$ l'algèbre des germes fonctions holomorphes dans un voisinage de $z_{\tau}$ dans $Z_{\tau}$.

Remarquons que l'homomorphisme composé $\mathbb{C}\left[U_{1}, \ldots, U_{s}\right] \rightarrow \mathbb{C}\left[S_{\tau}\right] \hookrightarrow \mathbb{C}\left[\left[S_{\tau}\right]\right]$ s'étend en un homomorphisme $\chi: \mathbb{C}\left\{U_{1}, \ldots, U_{s}\right\} \rightarrow \mathbb{C}\left[\left[S_{\tau}\right]\right]$ dont l'image est $\mathbb{C}\left\{S_{\tau}\right\}$. En effet, l'image du monôme $U^{\lambda}$, où $\lambda=\left(\lambda_{1}, \ldots, \lambda_{s}\right) \in \mathbb{N}^{s}$, est le monôme $X^{u(\lambda)}$, où $u(\lambda)=\sum \lambda_{i} u_{i} \in S_{\tau}$. Donc, l'image de $\phi$ est la série $\chi(\phi)=\sum_{u \in S_{\tau}}\left(\sum_{u(\lambda)=u} c_{\lambda}\right) X^{u}$, qui est bien définie parce que $\tau$ est strictement convexe. Supposons que $\phi$ est absolument convergente au point $x^{\prime}=\left(x_{1}^{\prime}, \ldots, x_{s}^{\prime}\right)$ correspondant au point $x \in Z_{\tau}$ par le plongement torique $Z_{\tau} \subset \mathbb{C}^{s}$. La valeur de la fonction $X^{u} \in \mathbb{C}\left[S_{\tau}\right]$ au point du $Z_{\tau}$, ne dépend pas de l'immersion, donc si $u=u(\lambda)$, on a $x(u)=x^{\prime \lambda}$ et la série $\chi(\phi)$ est absolument convergente au point $x$. Ceci implique que la série $\chi(\phi) \in \mathbb{C}\left[\left[S_{\tau}\right]\right]$ est convergente. Avec un raisonnement analogue, on peut montrer que l'homomorphisme d'algèbres $\chi: \mathbb{C}\left\{U_{1}, \ldots, U_{s}\right\} \rightarrow \mathbb{C}\left\{S_{\tau}\right\}$ est surjectif.

Par ailleurs, on a montré aussi que $\chi(\phi)$ est une fonction holomorphe dans un voisinage de $z_{\tau}$ dans $Z_{\tau}$, définissant un unique élément de $R$. Clairement, tous les éléments de $R$ sont obtenus de cette forme. Si la fonction $\chi(\phi)$ est nulle dans un voisinage de $z_{\tau}$ dans $Z_{\tau}$, la série $\phi$ est dans l'ideal engendré par $I$ dans $\mathbb{C}\left\{U_{1}, \ldots, U_{s}\right\}$ donc $\chi(\phi)=0$.

Comme conséquence de ce lemme, on déduit que l'anneau $\mathbb{C}\left\{S_{\tau}\right\}$ est noethérien et intégralement clos parce que $Z_{\tau}$ est une variété normale (voir [7, Section 71]).

\subsection{Extensions galoisiennes}

Soit $k \in \mathbb{Z}$ un entier positif fixé. Considérons les réseaux $N^{\prime}=k N \subset N$. Leurs réseaux duaux respectifs sont $M^{\prime}=\frac{1}{k} M \supset M$. Un cône $\tau$ strictement convexe dans $\left(\mathbb{R}^{d}\right)^{*}$ est rationnel pour les deux réseaux en même temps. Nous notons $Z_{\tau}$ (resp. $Z_{\tau}^{\prime}$ ) la variété torique associée à $(\tau, N)\left(\right.$ resp. à $\left.\left(\tau, N^{\prime}\right)\right)$. Le semi-groupe associé à $\left(\tau, N^{\prime}\right)$ est $S_{\tau}^{\prime}:=\frac{1}{k} S_{\tau} \subset$ $M^{\prime}$. L'homomorphisme de semi-groupes $M \supset S_{\tau} \hookrightarrow S_{\tau}^{\prime} \subset M^{\prime}$ définit un morphisme torique $f_{k}: Z_{\tau}^{\prime} \rightarrow Z_{\tau}$. L'image du point distingué de $Z_{\tau}^{\prime}$ est le point distingué du $Z_{\tau}$, donc on obtient un morphisme de germes irréductibles $\left(Z_{\tau}^{\prime}, z_{\tau}^{\prime}\right) \rightarrow\left(Z_{\tau}, z_{\tau}\right)$. En utilisant le lemme 1 on vérifie que l'homomorphisme des algèbres intègres associées est $\mathbb{C}\left\{S_{\tau}\right\} \hookrightarrow \mathbb{C}\left\{S_{\tau}^{\prime}\right\}$.

L'homomorphisme des semi-groupes $M \hookrightarrow M^{\prime}$ définit le morphisme $f_{k}: T^{\prime} \rightarrow T$ obtenu en restreignant $f_{k}$ aux tores respectifs de $Z_{\tau}^{\prime}$ et $Z_{\tau}$. On peut vérifier directement que 
le noyau de ce morphisme $f_{k \mid T^{\prime}}$, comme morphisme de groupes algébriques, est le sousgroupe fini $H$ de $T^{\prime}$, formé des éléments $\left(w_{1}, \ldots, w_{d}\right)$ tels que $w_{i}^{k}=1$, pour $i=1, \ldots, d$. Ce morphisme est un revêtement galoisien à $k^{d}$ feuilles de la variété $T$, parce que le groupe $H$ agit transitivement sur les fibres. Donc on a une extension galoisienne des corps des fonctions rationnelles $\mathbb{C}(T) \hookrightarrow \mathbb{C}\left(T^{\prime}\right)$. On va montrer qu'on a une situation analogue pour les corps des fonctions méromorphes aux points distingués des variétés toriques correspondantes.

Soit $L$ (resp. $L^{\prime}$ ) le corps des fractions de $\mathbb{C}\left\{S_{\tau}\right\}$, (resp. de $\mathbb{C}\left\{S_{\tau}^{\prime}\right\}$ ). L'homomorphisme $\mathbb{C}\left\{S_{\tau}\right\} \hookrightarrow \mathbb{C}\left\{S_{\tau}^{\prime}\right\}$ définit une extension de corps $L \hookrightarrow L^{\prime}$.

Lemme 2 L'extension de corps $L \hookrightarrow L^{\prime}$ est galoisienne. Soit $G$ son groupe de Galois. L'action de $H$ sur les monômes définit un épimorphisme de groupes $H \rightarrow G$ et l'ensemble des éléments G-invariants de l'anneau $\mathbb{C}\left\{S_{\tau}^{\prime}\right\}$ est $\mathbb{C}\left\{S_{\tau}\right\}$.

Preuve Clairement, $L \hookrightarrow L^{\prime}$ est une extension normale finie. À chaque $w \in H$ est associé l'homomorphisme d'algèbres $\mathbb{C}\left\{S_{\tau}^{\prime}\right\} \rightarrow \mathbb{C}\left\{S_{\tau}^{\prime}\right\}$ qui applique $X^{\frac{u}{k}} \mapsto w(u) X^{\frac{u}{k}}$. Cela définit un homomorphisme de groupes $H \rightarrow G$.

Remarquons que $X^{\frac{u}{k}} \mapsto w(u) X^{\frac{u}{k}}$ définit l'action de l'élément $w \in H$ sur un monôme de $\mathbb{C}\left[S_{\tau}^{\prime}\right]$. Le corollaire 1.16 de [10] garantit que le morphisme $Z_{\tau}^{\prime} \rightarrow Z_{\tau}$ coïncide avec la projection du quotient de $Z_{\tau}$ par rapport à l'action du groupe $H$. C'est-à-dire que $\mathbb{C}\left[S_{\tau}\right]$ est l'ensemble des éléments invariants de l'algèbre $\mathbb{C}\left[S_{\tau}^{\prime}\right]$ par l'action de $H$.

Si $H^{\prime}$ est l'image de $H$ dans $G$ on a montré que le sous-corps fixe de $L^{\prime}$ par $H^{\prime}$ coïncide avec $L$, donc $\left(L^{\prime}\right)^{G} \subset L$, c'est-à-dire que $L \subset L^{\prime}$ est une extension galoisienne et donc $H^{\prime}=G$.

\subsection{Paramétrisation de singularités quasi-ordinaires toriques}

Supposons que $F \in \mathbb{C}\left\{X_{1}, \ldots, X_{d}\right\}[Y]$ est un polynôme réduit tel que $0 \in \mathbb{C}$ est une racine de multiplicité $r \geq 1$ du polynôme $F(0, Y)$ et que le discriminant de $F$ soit de la forme $X^{q} \varepsilon$ où $\varepsilon$ est une unité de $\mathbb{C}\left\{X_{1}, \ldots, X_{d}\right\}$. D'après le théorème de préparation de Weierstrass, il existe un pseudo-polynôme à la Weierstrass $H$ de degré $r$ en $Y$, et une unité $\epsilon$ dans $\mathbb{C}\left\{X_{1}, \ldots, X_{d}, Y\right\}$ tels que $F=\epsilon H$. Par définition, la projection du germe $(\mathcal{X}, 0) \subset \mathbb{C}^{d} \times \mathbb{C}$ défini par le polynôme $H \in \mathbb{C}\left\{X_{1}, \ldots, X_{d}\right\}[Y]$ sur $\left(\mathbb{C}^{d}, 0\right)$ est quasi-ordinaire. D'après [1, Theorem 3], il existe $k \in \mathbb{N}$ tel que $H$ ait ses $r$ racines dans l'anneau $\mathbb{C}\left\{X_{1}^{1 / k}, \ldots, X_{d}^{1 / k}\right\}$.

On va généraliser la construction de racines associées à une projection quasi-ordinaire, au cas où le germe $\left(\mathbb{C}^{d}, 0\right)$ est remplacé par un germe de variété torique affine $\left(Z_{\tau}, z_{\tau}\right)$ au point distingué.

Théorème 1 Pour tout polynôme $F \in \mathbb{C}\left\{S_{\tau}\right\}[Y]$ réduit tel que le discriminant de $F$ soit de la forme $X^{u_{0}} \varepsilon$, où $\varepsilon$ est une unité dans l'anneau $\mathbb{C}\left\{S_{\tau}\right\}$ et que $0 \in \mathbb{C}$ soit une racine de multiplicité $r \geq 1$ du polynôme $F\left(z_{\tau}, Y\right)$ il existe $k \in \mathbb{N}$ tel que $F$ ait $r$ racines sans terme constant dans l'anneau $\mathbb{C}\left\{\frac{1}{k} S_{\tau}\right\}$.

Preuve Nous fixons un nombre fini de générateurs du semi-groupe $S_{\tau}$. Cela permet de définir un plongement de la variété torique affine $Z_{\tau} \subset \mathbb{C}^{s}$. Il lui est associé un épimor- 
phisme d'algèbres $\chi: \mathbb{C}\left\{U_{1}, \ldots, U_{s}\right\} \rightarrow \mathbb{C}\left\{S_{\tau}\right\}$ (voir le lemme 1). Considérons un polynôme $G \in \mathbb{C}\left\{U_{1}, \ldots, U_{s}\right\}[Y]$ tel que $G^{\chi}=F$. On a $G(0, Y)=F\left(z_{\tau}, Y\right)$. D'après le théorème de préparation de Weierstrass il existe un pseudo-polynôme à la Weierstrass $H$ de degré $r$ en $Y$, et une unité $\varepsilon$ dans $\mathbb{C}\left\{U_{1}, \ldots, U_{s}, Y\right\}$ tels que $G=\varepsilon H$. Clairement, les germes définis au point $\left(z_{\tau}, 0\right)$ par $F$ et par $H^{\chi}$ coïncident. Donc, on peut supposer que $F$ est un polynôme réduit de degré $r$ tel que $F\left(z_{\tau}, Y\right)=Y^{r}$.

Soit $L$ le corps de fractions de l'anneau intégre $\mathbb{C}\left\{S_{\tau}\right\}$. Les facteurs $F_{i}$ de la factorisation de $F$ en polynômes irréductibles dans $L[Y]$ sont dans $\mathbb{C}\left\{S_{\tau}\right\}[Y]$ parce que, d'après le lemme 1, l'anneau $\mathbb{C}\left\{S_{\tau}\right\}$ est intégralement clos et le coefficient de $Y^{r}$ est une unité (voir $\left[11\right.$, théorème 5 , section 3 , chap. V]). De plus, le discriminant de $F_{i}$ divise le discriminant de $F$ donc $\Delta_{Y} F_{i}$ est de la forme $X^{u} \epsilon$ où $\epsilon \in \mathbb{C}\left\{S_{\tau}\right\}$ est une unité. On peut donc supposer que $F$ est irréductible, engendrant un ideal premier $(F)$ dans $\mathbb{C}\left\{S_{\tau}\right\}[Y]$. Considérons le germe de variété analytique irréductible $(X, x) \subset\left(Z_{\tau} \times \mathbb{C}, x\right)$ au point $x$ correspondant à l'algèbre intègre $R=\mathbb{C}\left\{S_{\tau}\right\}[Y] /(F)$.

Soit $(X, x) \rightarrow\left(Z_{\tau}, z_{\tau}\right)$ la projection des germes, et choisissons un représentant fini $\pi: X \rightarrow Z_{\tau}$ tel que $\pi^{-1}\left(z_{\tau}\right)=\{x\}$. L'hypothèse sur le discriminant implique qu'il existe un voisinage $W$ du point $z_{\tau}$ dans $Z_{\tau}$ tel que $\pi$ est non ramifié sur $W^{*}:=W \cap T$. Par continuité, comme $\pi^{-1}\left(z_{\tau}\right)=\{x\}$, on peut supposer que $\pi^{-1}(W)$ est un sous-ensemble relativement compact de $\mathbb{C}^{s+1}$.

Comme $\pi$ est un morphisme fini, l'intersection de l'image inverse du lieu discriminant de $\pi$ avec $\mathcal{X}$ est une sous-variété analytique fermée propre de $\mathcal{X}$. Son complémentaire est un ouvert $X^{*} \subset X$, connexe parce que $X$ est analytiquement irréductible. Ceci montre que $\pi: X^{*} \rightarrow W^{*}$ est un revêtement connexe à $r$ feuillets.

On peut supposer que l'ouvert $W^{*}$ du tore $T$ est $\left.W^{*}=(\mathbb{D})^{*}\right)^{d}$ où $\left.\mathbb{D}\right)^{*}=D(0,1) \backslash\{0\} \subset$ $\mathbb{C}^{*}$. Soit $J$ le sous-groupe du groupe fondamental $\pi_{1}\left(W^{*}, w\right) \cong \mathbb{Z}^{d}$ associé au revêtement $\pi: X^{*} \rightarrow W^{*}$. Puisque $J$ est d'indice fini, il existe $k \in \mathbb{N}$ tel que $k Z^{d} \subset J$. Le revêtement $f_{k}: W^{*} \rightarrow W^{*}$, défini par $x \mapsto\left(x_{1}^{k}, \ldots, x_{d}^{k}\right)$, est associé au sous-groupe $k \mathbb{Z}^{d} \mathrm{du} \mathbb{Z}^{d}$. Donc, il existe un revêtement $p: W^{*} \rightarrow X^{*}$ tel que $\pi \circ p=f_{k}$, (voir [5, chap. 13]).

Clairement, $p$ est holomorphe, et borné dans le complémentaire dans $W$ d'un ensemble analytique fermé, c'est-à-dire que $p$ est une fonction faiblement holomorphe dans $W$. Comme $W \subset Z_{\tau}$ est une variété normale, toute fonction faiblement holomorphe est holomorphe, (voir [7, Section 71]). Donc, $p$ s'étend en un morphisme $W \rightarrow X$. La fonction holomorphe $\pi \circ p$ coïncide sur $W^{*}$ avec le morphisme torique $f_{k}: Z_{\tau}^{\prime} \rightarrow Z_{\tau}$ (où on considère $W^{*} \subset Z_{\tau}^{\prime}$ et aussi $W^{*} \subset Z_{\tau}$ ). Donc, elle est égale à la restriction du morphisme $f_{k}$ à $W$. Nous remarquons que $p\left(z_{\tau}^{\prime}\right)=x$ parce que $f_{k}\left(z_{\tau}^{\prime}\right)=z_{\tau}$ et $\pi^{-1}\left(z_{\tau}\right)=\{x\}$.

En utilisant le lemme 1, on voit que l'homomorphisme d'algèbres intègres associé au morphisme $f_{k}$ aux points distingués est $\mathbb{C}\left\{S_{\tau}\right\} \rightarrow \mathbb{C}\left\{S_{\tau}^{\prime}\right\}$. Considérons le monomorphisme d'algèbres $R \rightarrow \mathbb{C}\left\{S_{\tau}^{\prime}\right\}$ correspondant au morphisme de germes $p:\left(W, z_{\tau}^{\prime}\right) \rightarrow(X, x)$. L'algèbre $R$ est une sous- $\mathbb{C}\left\{S_{\tau}\right\}$-algèbre de $\mathbb{C}\left\{S_{\tau}^{\prime}\right\}$ parce que $\pi \circ p=f_{k}$. Nous avons donc un diagramme:

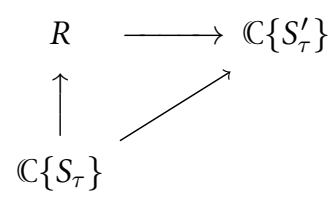

Soit $L$ (resp. $K, L^{\prime}$ ) le corps des fractions de $\mathbb{C}\left\{S_{\tau}\right\}$, (resp. de $R, \mathbb{C}\left\{S_{\tau}^{\prime}\right\}$ ). Par construction, 
$L \subset K \subset L^{\prime}$ et $K=L(\zeta)$ où $\zeta$ est l'image de $Y \in R$ dans $\mathbb{C}\left\{S_{\tau}^{\prime}\right\}$. D'après le lemme 2, l'extension de corps $L \hookrightarrow L^{\prime}$ est galoisienne, et la série $\zeta$ a ses $r$ conjugués dans l'anneau $\mathbb{C}\left\{S_{\tau}^{\prime}\right\}$. Ces conjugués sont les racines de $F$ dans $\mathbb{C}\left\{S_{\tau}^{\prime}\right\}$ qui paramétrisent $(X, x)$.

Remarque 1 Si $F$ est irréductible, on peut prendre $k=r$ dans le théorème 1.

En effet, puisque le polynôme $F$ est irréductible, l'indice du sous-groupe $J$ (dans la preuve du théorème 1), est égal à $r$. L'ordre du sous-groupe engendré par l'image d'un vecteur de la base canonique de $\mathbb{Z}^{d}$ dans $\mathbb{Z}^{d} / J$ est un diviseur de $r$. Donc, on a $r \mathbb{Z}^{d} \subset J$.

\section{Racines à la Newton Puiseux}

\subsection{Valuation induite par un vecteur irrationnel}

Soit $R$ un anneau commutatif et $\Gamma$ un groupe totalement ordonné. Une valuation $\omega$ de $R$ dans $\Gamma$ est une application $\omega: R \backslash\{0\} \rightarrow \Gamma$ tel que

(i) $\omega(a b)=\omega(a)+\omega(b)$ pour $0 \neq a, b \in R$,

(ii) $\omega(a-b) \geq \inf (\omega(a), \omega(b))$ pour $0 \neq a, b \in R$ et $a \neq b$, avec égalité si $\omega(a) \neq \omega(b)$.

On associe à chaque $\lambda \in \Gamma$ l'ensemble $\mathcal{J}_{\lambda}=\{a \in R / \omega(a)>\lambda\}$. L'ensemble $\mathcal{J}_{\lambda}$ est un ideal de $R$ et on a $\lambda>\beta \Rightarrow \mathcal{J}_{\lambda} \subset \mathcal{J}_{\beta}$. La topologie $\omega$-adique sur $R$, est la topologie qui fait de $R$ un groupe topologique dans lequelle l'ensemble des ideaux $\left\{\mathcal{J}_{\lambda}\right\}_{\lambda \in \Gamma}$ est un système fondamental de voisinages de $0 \in R$.

La valuation $\omega$ est archimédienne si $\Gamma$ est isomorphe comme groupe totalement ordonné à un sous-groupe de $\mathbb{R}$. Soit $(R, \mathfrak{M})$ est un anneau local de corps de fractions $L$. La valuation $\omega$ de $L$ est centrée sur $R$ si $\omega(a) \geq 0$ pour $a \in R$ et $\omega(a)>0$ pour $a \in \mathfrak{M}$.

Lemme 3 Soient $(R, \mathfrak{M})$ un anneau local noethérien de corps de fractions $L$, et $\omega$ une valuation archimédienne de $L$ centrée sur $R$. Alors, la topologie $\mathfrak{M}$-adique coüncide avec la topologie $\omega$-adique de $R$.

Preuve Puisque $R$ est noethérien, le semi-groupe $\omega(R \backslash\{0\})$ est bien ordonné, et il existe un plus petit élément $\lambda$ de l'ensemble $\omega(\mathfrak{M} \backslash\{0\})$. Si $\beta \in \omega(\mathfrak{M} \backslash\{0\})$, il existe $n \in \mathbb{N}$ tel que $n \lambda>\beta$ donc $\mathfrak{M}^{n} \subset \mathcal{J}_{\beta} \subset \mathfrak{M}$.

On appelle un vecteur $w \in \mathbb{R}^{d}$ irrationnel si ses coordonneés sont linéairement indépendantes sur $\left(\mathbb{Q}\right.$. Associé à un vecteur irrationnel $w \in \mathbb{R}^{d}$ nous définissons un ordre total sur $\mathbb{Q}^{d}{ }^{d}$ par:

$$
u<_{w} u^{\prime} \Leftrightarrow\langle u, w\rangle<\left\langle u^{\prime}, w\right\rangle .
$$

Remarque 2 Soit $\tau$ un cône rationnel strictement convexe de dimension $d$ dans $\mathbb{R}^{d}$. Un vecteur irrationnel $w \in \tau^{\vee}$ définit une valuation archimédienne de l'anneau local complet $\mathbb{C}\left[\left[S_{\tau}\right]\right]$ par $w\left(\sum_{u \in S_{\tau}} c_{u} X^{u}\right)=\min _{\mathcal{c}_{u} \neq 0}(\langle u, w\rangle)$. Cette valuation vérifie les hypothèses du lemme 3.

Si $\left(\phi_{j}\right) \subset \mathbb{C}\left[\left[S_{\tau}\right]\right]$ vérifie que la suite $\left(w\left(\phi_{j}\right)\right) \subset \mathbb{R}$ est strictement croissante alors $\phi_{j}$ tend vers $0 \in \mathbb{C}\left[\left[S_{\tau}\right]\right]$. 
On appelle l'exposant initial d'une série $\phi \in \mathbb{C}\left[\left[S_{\tau}\right]\right]$ par rapport a $w$, l'exposant $u$ de $\phi$ tel que $w\left(X^{u}\right)=w(\phi)$. L'exposant initial est le plus petit, pour l'ordre $<_{w}$, parmi les exposants de $\phi$.

\subsection{Chemins monotones dans le polyèdre de Newton}

Un polyèdre $\mathcal{N}$ dans $\mathbb{R}^{d}$ est l'intersection d'une famille de demi-espaces d'équation $\langle\omega, u\rangle \geq$ $\lambda_{\omega}$, pour $\omega \in \Xi \subset\left(\mathbb{R}^{d}\right)^{*}$. On dira que le polyèdre $\mathcal{N}$ est rationnel si ses sommets sont dans le réseau $\mathbb{Z}^{d}$ et si ses faces ont des équations à coefficients dans $\mathbb{O}$. Le cône $\tau$ associé au sommet $u$ d'un polyèdre rationnel $\mathcal{N}$ dans $\mathbb{R}^{d}$ est l'ensemble des fonctions linéaires qui atteignent leur valeur minimale sur $\mathcal{N}$ au sommet $u$. Le cône $\tau$ est rationnel de dimension $d$, et $\tau$ est strictement convexe si et seulement si le polyèdre $\mathcal{N}$ est de dimension $d$. Dans ce cas, l'ensemble des cônes associés au polyèdre $\mathcal{N}$ forme un éventail $\Sigma$ dans $\left(\mathbb{R}^{d}\right)^{*}$ avec un nombre éventuellement infini de cônes; le support $|\Sigma|=\bigcup_{\sigma \in \Sigma} \sigma$ de l'éventail associé à $\mathcal{N}$ n'est pas nécessairement fermé. Si $\tau \subset|\Sigma|$ est un cône rationnel strictement convexe de dimension $d$, l'ensemble des cônes $\tau \cap \sigma$, pour $\sigma \in \Sigma$ est une subdivision de $\tau$. En particulier, c'est l'éventail associé à la somme de Minkowski $\tau^{\vee}+\mathcal{N}$. Cette subdivision est finie parce que si $S^{d-1}$ est la sphère unité $\left\{\tau \cap \sigma \cap S^{d-1}\right\}$ est un complexe polyèdral de support l'ensemble compact $\tau \cap \sigma \cap S^{d-1}$.

Considérons $\mathbb{R}^{d} \times \mathbb{R}$ avec des coordonnées fixées $(u, v)$. On dira qu'une arête bornée $e$ d'un polyèdre $\mathcal{N} \subset \mathbb{R}^{d} \times \mathbb{R}$ est admissible si elle n'est pas parallèle à l'hyperplan $v=0$. Une arête admissible est de la forme $\left[p_{v_{1}}, p_{v_{2}}\right]$ où $p_{v_{i}}=\left(u_{v_{i}}, v_{i}\right) \in \mathbb{R}^{d} \times \mathbb{R}$ avec $v_{1}<v_{2}$. Nous appellerons le vecteur $q_{e}:=\frac{u_{v_{1}}-u_{v_{2}}}{v_{2}-v_{1}}$ l'inclinaison, et le nombre $l_{e}=v_{2}-v_{1} \in \mathbb{N}$ la longueur de l'arête. Considérons la projection $\pi_{e}: \mathbb{R}^{d} \times \mathbb{R}^{\mathbb{R}} \rightarrow \mathbb{R}^{d} \times\{0\}$ parallèlement à l'arête $e$, définie par $\pi_{e}(u, v)=u+v q_{e}$. Le cône $\sigma(e) \subset\left(\mathbb{R}^{d}\right)^{*}$, associé au sommet $\pi_{e}(e)$ du polyèdre $\pi_{e}(\mathcal{N})$ et de dimension $d$ et on a:

Lemme 4 Pour $w \in\left(\mathbb{R}^{d}\right)^{*}$, les propriétés suivantes sont équivalentes:

1. $w \in \sigma(e)$.

2. La fonction lineaire $w$ atteint sa valeur minimale sur chaque section $v=\lambda$ de $\mathcal{N}$ au point $(u(\lambda), \lambda)$ de l'arête $e$.

On dit qu'un chemin $\gamma$ dans les arêtes de $\mathcal{N} \subset \mathbb{R}^{d} \times \mathbb{R}$ est monotone si on peut le paramétriser par $\gamma(\lambda)=(u(\lambda), \lambda)$. Supposons que le chemin $\gamma$ a pour sommets $\left\{p_{0}, p_{i_{1}}, \ldots\right.$, $\left.p_{i_{t}}, p_{n}\right\}$ avec $p_{j}=\left(u_{j}, j\right)$ pour $j \in\left\{i_{0}, i_{1}, \ldots, i_{t}, i_{t+1}\right\}$ avec $0=i_{0}<\cdots<i_{t+1}=n$. Nous notons $q_{r}$ l'inclinaison du segment $e_{r}=\left[p_{i_{r}}, p_{i_{r+1}}\right]$, pour $r=0, \ldots, t$. Le chemin monotone $\gamma$ est cohérent si il existe $w \in\left(\mathbb{R}^{d}\right)^{*}$ tel que $\gamma(\lambda)$ est l'unique point de la section $v=\lambda$ du polyèdre $\mathcal{N}$ en lequel $w$ atteint sa valeur minimale sur cette section, pour $\lambda \in[0, n]$.

Lemme 5 Avec les notations précedentes, si $w \in\left(\mathbb{R}^{d}\right)^{*}$ est un vecteur irrationnel définissant le chemin monotone cohérent $\gamma$ dans les arêtes $d u$ polyèdre rationnel $\mathcal{N}$, alors les inclinaisons des arêtes de $\gamma$ vérifient:

$$
q_{t}<_{w} q_{t-1}<_{w} \cdots<_{w} q_{0}
$$


Preuve Notons $\pi_{s}$ la projection parallèlement au segment $e_{s}$. Le vecteur $\pi_{s-1}\left(e_{s-1}\right)-\pi_{s}\left(e_{s}\right)$ a le même sens que le vecteur $\pi_{s}\left(p_{i_{s-1}}\right)-\pi_{s}\left(e_{s}\right)$. Par le lemme précedent $w$ appartient à $\sigma\left(e_{s}\right)$, donc on a $\left\langle w, \pi_{s-1}\left(e_{s-1}\right)-\pi_{s}\left(e_{s}\right)\right\rangle=i_{s}\left\langle w, q_{s-1}-q_{s}\right\rangle \geq 0$, pour $s=1, \ldots, t$.

Soit $\rho \subset\left(\mathbb{R}^{d}\right)^{*}$ un cône strictement convexe de dimension $d$. Nous notons $\mathbb{C}\left(\left(S_{\rho}\right)\right)$ (resp. $\mathbb{C}\left\{\left\{S_{\rho}\right\}\right\}$ ) l'anneau de fractions de l'anneau $\mathbb{C}\left[\left[S_{\rho}\right]\right]$ (resp. $\mathbb{C}\left\{S_{\rho}\right\}$ ) pour l'ensemble multiplicativement fermé correspondant aux monômes $X^{u}$ pour $u \in S_{\rho}$.

Définition 1 Le $\rho$-polyèdre de Newton d'une série $\phi \in \mathbb{C}\left(\left(S_{\rho}\right)\right)$ non nulle est la somme de Minkowski de l'enveloppe convexe de ses exposants et du cône $\rho^{\vee}$. Le $\rho$-polyèdre de Newton d'un polynôme $F \in \mathbb{C}\left(\left(S_{\rho}\right)\right)[Y]$, est la somme de Minkowski de l'enveloppe convexe de ses exposants et du cône $\rho^{\vee} \times\{0\}$.

Le $\rho$-polyèdre de Newton de $\phi$, que nous notons $\mathcal{N}_{\rho}(\phi)$, est un polyèdre de dimension $d$ ayant un nombre fini de sommets. L'éventail associé est la subdivision du cône $\rho$ induite par l'éventail associé à l'enveloppe convexe des exposants de $\phi$.

Nous notons $\mathcal{N}_{\rho}(F)$ le polyèdre de Newton d'un polynôme $F \in \mathbb{C}\left(\left(S_{\rho}\right)\right)[Y]$. Remarquons que le $\rho$-polyèdre de Newton de $F$ ne dépend que des exposants de $F$, il dépend aussi de l'anneau dans lequel on considère que se trouvent les coefficients de $F$. Pour tout cône $\tau \subset\left(\mathbb{R}^{d}\right)^{*}$ rationnel strictement convexe de dimension $d$, l'inclusion d'algèbres $\mathbb{C}\left[X_{1}, \ldots, X_{d}\right] \rightarrow \mathbb{C}\left\{\left\{S_{\tau}\right\}\right\}$, permet de considérer un polynôme $F \in \mathbb{C}\left[X_{1}, \ldots, X_{d}\right][Y]$ comme élement de $\mathbb{C}\left\{\left\{S_{\tau}\right\}\right\}[Y]$. L'enveloppe convexe des exposants de $F$ est un polyèdre compact, $\mathcal{P}(F)$, mais le polyèdre $\mathcal{N}_{\rho}(F)$ n'est pas compact.

On définit la relation suivante parmi les vecteur irrationnels du cône $\rho: w \sim w^{\prime}$, si et seulement si, ils définissent le même chemin polygonal dans les arêtes du polyèdre $\mathcal{N}_{\rho}(F)$. Par le lemme 4, cette relation définit un éventail qui subdivise le cône $\rho$. Cet éventail est défini par un polyédre que nous allons décrire maintenant.

\subsection{Le polyèdre-fibre de la projection du polyèdre de Newton}

Soient $\mathcal{P} \subseteq \mathbb{R}^{N}$ un polytope et $\pi: \mathbb{R}^{N} \rightarrow \mathbb{R}^{M}$ une application affine surjective, l'image de $\mathcal{P}$ est un polytope $\mathcal{Q}$. L'intégrale de Minkowski de l'application $\pi: \mathcal{P} \rightarrow \mathcal{Q}$ est l'ensemble des intégrales $\int_{Q} \gamma \in \mathbb{R}^{N}$ lorsque $\gamma$ parcourt l'ensemble des sections Borel-mesurables $\gamma: \mathcal{Q} \rightarrow$ $\mathcal{P}$ de $\pi$. D'après [3], l'intégrale de Minkowski est un polytope convexe de dimension égale à $\operatorname{dim} \mathcal{P}-\operatorname{dim} Q$.

Si $F$ est un polynôme dans $\mathbb{C}\left[X_{1}, \ldots, X_{d}\right][Y]$, son polytope de Newton $\mathcal{P}(F) \subset \mathbb{R}^{d+1}$ est l'enveloppe convexe de ses exposants. Considérons un polynôme de la forme $F=$ $\sum_{k=0}^{n} a_{k} Y^{k}$, où les $a_{k}$ sont des polynômes dans $\mathbb{C}\left[X_{1}, \ldots, X_{d}\right]$ avec $a_{0} a_{n} \neq 0$. Soit $\mathcal{P}(F)$ le polytope de Newton de $F$; nous allons décrire l'intégrale de Minkowski de la projection $\pi: \mathcal{P}(F) \subset \mathbb{R}^{d} \times \mathbb{R} \rightarrow[0, n] \subset \mathbb{R}$. Ceci est un cas particulier du théorème 7.3 de [3]. Une section de $\pi$ est une application monotone de la forme $t \mapsto(\gamma(t), t) \in \mathcal{P}(F)$, pour $t \in[0, n]$, et il lui est associé le point $\int_{[0, n]} \gamma$ dans l'intégrale de Minkowski de $\pi$. Il est montré en [3] que les sommets de l'intégrale de Minkowski correspondent à des intégrales des chemins monotones cohérents dans les arêtes de $\mathcal{P}(F)$.

Ces chemins sont décrits par une collection $\left\{p_{0}, p_{i_{1}}, \ldots, p_{i_{s}}, p_{n}\right\}$ de sommets de $\mathcal{P}(F)$, avec $p_{j}=\left(u_{j}, j\right)$, où $u_{j}$ est un sommet de $\mathcal{P}\left(a_{j}\right)$ pour $j \in\left\{0, i_{1}, \ldots, i_{s}, n\right\}$, avec $0<i_{1}<$ 
$\cdots<i_{s}<n$. Si $v<v^{\prime}$ on peut paramétriser le segment $\left[(u, v),\left(u^{\prime}, v^{\prime}\right)\right] \subset \mathbb{R}^{d} \times \mathbb{R}$, par $\gamma(\lambda)=u+\frac{\lambda-v}{v^{\prime}-v}\left(u^{\prime}-u\right)$, avec $\lambda \in\left[v, v^{\prime}\right]$, donc $\int_{\left[v, v^{\prime}\right]} \gamma=\frac{v^{\prime}-v}{2}\left(u+u^{\prime}\right)$.

L'intégrale du chemin $\gamma$ correspondant à la collection de sommets $\left\{p_{0}, p_{i_{1}}, \ldots, p_{i_{s}}, p_{n}\right\}$ est:

$$
\begin{aligned}
\int_{[0, n]} \gamma & =\int_{\left[0, i_{1}\right]} \gamma+\cdots+\int_{\left[i_{s}, n\right]} \gamma \\
& =\frac{1}{2}\left(i_{1}\left(u_{0}+u_{i_{1}}\right)+\sum_{k=2}^{s}\left(i_{k}-i_{k-1}\right)\left(u_{i_{k}}+u_{i_{k-1}}\right)+\left(n-i_{s}\right)\left(u_{i_{s}}+u_{n}\right)\right) \\
& =\frac{1}{2}\left(i_{1} u_{0}+i_{2} u_{i_{1}}+\sum_{k=2}^{s-1}\left(i_{k+1}-i_{k-1}\right) u_{i_{k}}+\left(n-i_{s-1}\right) u_{i_{s}}+\left(n-i_{s}\right) u_{n}\right) .
\end{aligned}
$$

Ces considérations motivent la définition suivante:

Définition 2 Soient un cône strictement convexe $\rho \subset \mathbb{R}^{d}$ de dimension $d$ et $F \in$ $\mathbb{C}\left(\left(S_{\rho}\right)\right)[Y]$ un polynôme de degré $n$ de terme constant non nul. Soit $Q$ l'enveloppe convexe des intégrales $\int \gamma_{w}$ des chemins monotones $\gamma$ dans le polyèdre $\mathcal{N}_{\rho}(F)$ définis par des vecteurs irrationnels $w \in \rho$. Le $\rho$-polyèdre-fibre de $F$ est la somme de Minkowski $Q_{\rho}(F):=$ $2\left(Q+\rho^{\vee}\right)$.

Le $\rho$-polyèdre-fibre $Q_{\rho}(F)$ est un polyèdre rationnel. Il dépend du $\rho$-polyèdre de Newton de $F$. L'éventail $\Sigma$ associé au polyèdre-fibre $Q_{\rho}(F)$ est une subdivision rationnelle finie du cône $\rho$. Si $w, w^{\prime}$ sont des vecteurs dans l'interieur d'un cône de dimension $d$ de $\Sigma$, ils définissent le même chemin polygonal dans les arêtes du polyèdre $\mathcal{N}_{\rho}(F)$.

Dans le cas où $F$ est un polynôme dans l'anneau $\mathbb{C}\left[X_{1}, \ldots, X_{d}\right][Y]$ on appelle polytopefibre de $F$ le polytope $Q(F):=2 \mathcal{Q}$ où $Q$ est l'intégrale de Minkowski de la la projection du polytope de Newton $\pi: \mathcal{P}(F) \subset \mathbb{R}^{d} \times \mathbb{R} \rightarrow[0, n] \subset \mathbb{R}$.

\subsection{Théorème de Newton-Puiseux}

On va généraliser un résultat de [9].

Théorème 2 Soient $\rho$ un cône rationnel strictement convexe de dimension d et $F \in$ $\mathbb{C}\left(\left(S_{\rho}\right)\right)[Y]$ un polynôme non constant. Pour tout vecteur irrationnel $w \in \rho$ il existe un cône rationnel strictement convexe $\sigma_{w}$ de dimension $d$, et $k \in \mathbb{N}$ tels que $w \in \sigma_{w} \subset \rho$ et que $F$ se décompose dans l'anneau $\mathbb{C}\left(\left(\frac{1}{k} S_{\sigma_{w}}\right)\right)[Y]$.

Preuve Elle est essentiellement la même que celle de [9]. Un vecteur irrationnel $w \in \rho \subset$ $\left(\mathbb{R}^{d}\right)^{*}$ définit un chemin monotone cohérent $\gamma$ dans les arêtes du polyèdre rationnel $\mathcal{N}_{\rho}(F)$. Fixons une arête $e=\left[(u, v),\left(u^{\prime}, v^{\prime}\right)\right] \subset \mathbb{R}^{d} \times \mathbb{R}$ du chemin $\gamma$, avec $v<v^{\prime}$. L'inclinaison de $e$ est un vecteur $q \in \frac{1}{l} Z^{d}$ où $l$ est la longueur $v^{\prime}-v$. La restriction de $F$ à l'arête $e$ est le polynôme $F_{\mid e}=\sum_{I \in e} \alpha_{I} X_{1}^{i_{1}} \cdots X_{d}^{i_{d}} Y^{i_{d+1}}$. On associe à l'arête $e$ le polynôme $f_{e} \in \mathbb{C}[t]$ par $F_{\mid e}(1, \ldots, 1, t)=t^{v} f_{e}$ où $f_{e}(0) \neq 0$. Le polynôme $f_{e}$ est de degré $l$ et toutes ses racines sont non nulles. 
Soit $c$ une racine de $f_{e}$, définissons le polynôme $F_{2}=F\left(Y+c X^{q}\right) \in\left(\mathbb{C}\left(\left(\frac{1}{l} S_{\rho}\right)\right)[Y]\right.$. Clairement, $F_{2}$ est un polynôme de degré $r$ et on a:

$$
F_{2}=\sum_{I} \alpha_{I} \sum_{j=0}^{i_{d+1}}\left(\begin{array}{c}
i_{d+1} \\
j
\end{array}\right) c^{j} X_{1}^{i_{1}+j q_{1}} \cdots X_{d}^{i_{d}+j q_{d}} Y^{i_{d+1}-j} .
$$

On en déduit que:

1. Les exposants de $F_{2}$ sont de la forme $I+j(q,-1)$ où $I=\left(i_{1}, \ldots, i_{d+1}\right)$ est un exposant de $F$ et $j \in\left\{0, \ldots, i_{d+1}\right\}$, donc $\pi_{e}\left(\mathcal{N}_{\rho}\left(F_{2}\right)\right)$ est contenu dans $\pi_{e}\left(\mathcal{N}_{\rho}(F)\right)$.

2. Le coefficient du terme constant de $F_{2}$ d'exposant dans la droite $E$ défini par l'arête $e$ est, $\sum_{I \in e} \alpha_{I} c^{i_{d+1}}=f_{e}(c)$, nul par construction.

3. Le coefficient du terme de $F_{2}$ d'exposant $p_{v^{\prime}}$ coïncide avec celui de $F$.

4. Si $Y$ ne divise pas $F_{2}$, le polyèdre de $F_{2}$ a toujours des points dans l'hyperplan $v=0$.

5. L'exposant de $F_{2}$ dans la droite $E$ correspondant au terme de plus petit degré en $Y$ est un sommet du $\rho$-polyèdre de Newton de $F_{2}$. Cet exposant est de la forme $(u, m)$ où $m$ est la multiplicité de $c$ comme racine de $f_{e}$. En effet, la plus petite ordonnée des exposants de $F_{2}$ dans la droite $E$ est le nombre $m$ de fois qu'il faut dériver pour que $\frac{\partial^{m}}{\partial Y^{m}}\left(F_{2} \mid E\right)$, ait un terme constant non nul. Comme $F_{2 \mid E}=F_{\mid e}\left(Y+c X^{q}\right)$ le coefficient du terme constant de $Y$ de $\frac{\partial^{k}}{\partial Y^{k}}\left(F_{2 \mid E}\right)$ est égal à $\sum_{I \in e} \alpha_{I} i_{d+1} \cdots\left(i_{d+1}-k+1\right) c^{i_{d+1}-k}=\frac{d^{k} f_{e}}{d t^{k}}(c)$.

Le sommet $(u, m)$ du polyèdre $\mathcal{N}_{\rho}\left(F_{2}\right)$ défini par 5 . est un sommet du chemin monotone défini par $w$ dans les arêtes de $\mathcal{N}_{\rho}\left(F_{2}\right)$. Si $Y$ ne divise pas $F_{2}$ on va considérer la partie finale du chemin entre le sommet $(u, m)$ et l'hyperplan $v=0$.

Parmi les segments de cette partie finale du chemin polygonal on choisit une arête $e_{2}$ d'inclinaison $q_{2}$ et de longueur $l_{2}$. On choisit une racine $c_{2}$ du polynôme associé $f_{e_{2}}$ de multiplicité $m_{2}$ et on définit $F_{3}:=F_{2}\left(Y+c_{2} X^{q_{2}}\right)$. On continue par récurrence. Le polynôme $F_{n}$ est un élément de l'anneau $\mathbb{C}\left(\left(\frac{1}{l_{1} \cdots l_{n-1}} S_{\rho}\right)\right)[Y]$.

On obtient une suite décroissante de nombres entiers positifs: $l \geq m \geq l_{2} \geq m_{2}>$ $\cdots>0$, qui est donc stationnaire; il existe $n_{0} \in \mathbb{N}$ tel que pour tout $n \geq n_{0}$ on a $l_{n}=m_{n}=$ $m_{n_{0}}=m$. Ceci implique que $f_{e_{n}}=\theta\left(t-c_{n}\right)^{m}$, et aussi que la partie finale du chemin défini par $w$ dans les arêtes de $\mathcal{N}_{\rho}\left(F_{n}\right)$ est le segment $e_{n}$. De plus les sommets de $e_{n}$ et $e_{n+1}$ qui ne sont pas dans l'hyperplan $v=0$ coïncident, pour $n>n_{0}$.

Pour $n>n_{0}$, on a le segment $e_{n}=\left[\left(u_{n}, 0\right),\left(u_{0}, m\right)\right]$ d'inclinaison $q_{n}=\frac{1}{m}\left(u_{n}-u_{0}\right)$. L'intersection de la droite définie par $e_{n}$ avec l'hyperplan $v=0$ est le point $p_{n}:=u_{0}+m q_{n}$. Par définition du $\rho$-polyèdre de Newton, le cône $\sigma\left(e_{n_{0}}\right)$ associé au sommet $p_{n_{0}}$ du polyèdre $\pi_{e_{n_{0}}}\left(\mathcal{N}_{\rho}\left(F_{n_{0}}\right)\right)$ est contenu dans $\rho$. Le lemme 4 implique que $w \in \sigma\left(e_{n_{0}}\right)$.

On vérifie qu'il existe $k \in \mathbb{N}$ tel que les inclinaisons construites sont dans un réseau $\frac{1}{k} \mathbb{Z}^{d}$. On sait que $u_{0}=\frac{\beta_{0}}{k}, u_{n_{0}}=\frac{\beta_{n_{0}}}{k}$ où $\beta_{0}, \beta_{n_{0}} \in \mathbb{Z}^{d}$ et $k=l_{1} l_{2} \cdots l_{n_{0}-1}$ est un entier. L'inclinaison de $e_{n_{0}}$ est $q_{n_{0}}=\frac{\beta_{n_{0}}-\beta_{0}}{k m}=\frac{\beta}{k \lambda}$ où $\beta \in \mathbb{Z}^{d}$ et $\lambda \in \mathbb{N}$ est premier avec une coordonnée de $\beta$. Si $\left(\frac{\beta^{\prime}}{k}, h\right)$ est un exposant de $F_{\mid e}^{n_{0}}$ on a $q_{n_{0}}=\frac{\beta_{n_{0}}-\beta^{\prime}}{k h}=\frac{\beta}{k \lambda}$. Comme $\lambda\left(\beta_{n_{0}}-\beta^{\prime}\right)=h \beta$, on déduit que $\lambda$ divise $h$ donc $f_{e_{n_{0}}}$ est un polynôme en $t^{\lambda}$. Par ailleurs $f_{e_{n}}=\theta\left(t-c_{n}\right)^{m}$ et comme la caractéristique de $\mathbb{C}$ est zéro, on a $\lambda=1$. Par récurrence on obtient que $q_{n} \in \frac{1}{k} \mathbb{Z}^{d}$, pour $n \geq n_{0}$ et donc pour $n \in \mathbb{N}$.

Montrons que les inclinaisons $q_{j}$ sont dans un cône affine strictement convexe. 
Le $\rho$-polyèdre de Newton de $F_{n_{0}}$ est contenu dans le cone affine:

$$
W\left(e_{n_{0}}\right):=\left\{\lambda\left(u-u^{\prime}\right) / u \in \mathcal{N}_{\rho}\left(F_{n_{0}}\right), u^{\prime} \in e_{n_{0}}, \lambda \geq 0\right\}
$$

associé à l'arête $e_{n_{0}}$. Comme $u_{n_{0}+1} \in W\left(e_{n_{0}}\right)$, par construction on a l'inclusion $\mathcal{N}_{\rho}\left(F_{n_{0}+1}\right) \subset$ $W\left(e_{n_{0}}\right)$. Par récurrence, en utilisant que le sommet $\left(u_{0}, m\right)$ de $e_{n}$ est sur la droite qui contient le segment $e_{n_{0}}$, on montre que $u_{n} \in W\left(e_{n_{0}}\right)$ et que $\mathcal{N}_{\rho}\left(F_{n}\right) \subset W\left(e_{n_{0}}\right)$, pour $n>n_{0}$. Ceci implique pour tout $w^{\prime} \in \sigma\left(e_{n_{0}}\right)$ que $\left\langle w^{\prime}, q_{n}-q_{n_{0}}\right\rangle=\frac{1}{m}\left\langle w^{\prime}, p_{n}-p_{n_{0}}\right\rangle$ est $\geq 0$. Donc les exposants construits sont dans le cône rationnel affine $q_{n_{0}}+\sigma\left(e_{n_{0}}\right)^{\vee}$ pour $n \geq n_{0}$.

Notons $\sigma$ pour $\sigma\left(e_{n_{0}}\right)$. Il existe $u_{0} \in \frac{1}{k} \mathbb{Z}^{d}$ tel que les inclinaisons $q_{n}$ appartiennent à $u_{0}+\frac{1}{k} S_{\sigma}$. Définissons les sommes partielles, $\phi_{n}=\sum_{j=1}^{n} c_{j} X^{q_{j}}$ pour $n \in \mathbb{N}$. On a $\phi_{n} \in$ $\mathbb{C}\left(\left(\frac{1}{k} S_{\sigma}\right)\right)$ et $X^{-u_{0}} \phi_{n} \in \mathbb{C}\left[\left[\frac{1}{k} S_{\sigma}\right]\right]$. Par construction, et par le lemme 5 , on sait que $q_{j}<_{w} q_{j+1}$ pour $j \in \mathbb{N}$. Par la remarque 2, ceci implique que la série formelle $\phi:=\sum c_{j} X^{q_{j}}$ est égale à $X^{u_{0}} \lim _{n \rightarrow \infty} X^{-u_{0}} \phi_{n}$ où la limite est dans l'anneau complet $\mathbb{C}\left[\left[\frac{1}{k} S_{\sigma}\right]\right]$.

Comme $w \in \sigma \subset \rho$, on peut considérer $F$ comme élément de $\mathbb{C}\left(\left(\frac{1}{k} S_{\sigma}\right)\right)[Y]$. La série formelle $\phi$ est une racine de $F$. En effet, si $n \geq n_{0}$ la série $F\left(\phi_{n-1}\right)=F_{n}(0)$ a tous ses exposants dans le cône rationnel affine $p_{n_{0}}+\sigma\left(e_{n_{0}}\right)^{\vee}$. L'égalité suivante $F(\phi)=$ $X^{p_{n_{0}}} \lim _{n \rightarrow \infty} F\left(\phi_{n-1}\right) X^{-p_{n_{0}}}$ est clair. Si $n \geq n_{0}$ l'exposant initial de $F\left(\phi_{n-1}\right) X^{-p_{n_{0}}}$ par rapport à $w$ est $p_{n+1}-p_{n}=m\left(q_{n+1}-q_{n_{0}}\right)$ et on a $\lim _{n \rightarrow \infty} F_{n}(0) X^{-p_{n_{0}}}=0$.

On vérifie que la multiplicité de $\phi$ comme racine de $F$ est $\geq m$. La multiplicité de $c_{n}$ comme racine de $f_{e_{n}}$ est $\geq m$, donc $c_{n}$ est une racine de $\frac{d^{s} f_{e_{n}}}{d t^{k}}$ pour $1 \leq s \leq m-1$. Le polynôme $\frac{d^{s} f_{e_{n}}}{d t^{s}}$ est le polynôme de l'arête $e_{n}^{s}$ du polyèdre $\mathcal{N}_{\rho}\left(\frac{\partial^{s} F}{\partial Y^{s}}\right)$ qui est sur le segment $-(0, \ldots, 0, s)+e_{n}$. L'arête $e_{n}^{s}$ est déterminée par le vecteur irrationnel $w$. Comme $\frac{\partial^{s} F_{n}}{\partial Y^{s}}=$ $\frac{\partial^{s} F}{\partial Y^{s}}\left(Y+\phi_{n-1}\right)$ on obtient que $\phi$ est une racine de $\frac{\partial^{s} F}{\partial Y^{s}}$ pour $1 \leq s \leq m-1$.

On a montré que, associés à chaque arête $e$ du chemin monotone $\gamma$, il existe $k \in \mathbb{N}$ et un cône rationnel $\sigma_{e}$ strictement convexe de dimension $d$ tel que $w \in \sigma_{e} \subset \rho$, tels que $F$ ait au moins $l_{e}$ racines à la Newton-Puiseux dans $\mathbb{C}\left[\left[\frac{1}{k} S_{\sigma_{e}}\right]\right]$. On peut choisir $k \in \mathbb{N}$ valable pour toutes les arêtes de $\gamma$. Comme le vecteur $w$ est irrationnel, le cône rationnel $\tau=$ $\bigcap_{e \in \gamma} \sigma_{e}$ est de dimension $d$. L'existence d'un homomorphisme d'algèbres $\mathbb{C}\left[\left[\frac{1}{k} S_{\sigma_{e}}\right]\right] \hookrightarrow$ $\mathbb{C}\left[\left[\frac{1}{k} S_{\tau}\right]\right]$, pour chaque arête $e$ de $\gamma$, garantit que $F$ se décompose dans $\mathbb{C}\left[\left[\frac{1}{k^{\prime}} S_{\tau}\right]\right]$, parce que les exposants initiaux par rapport à $<_{w}$ des séries correspondantes à segments différents de $\gamma$ sont différents.

Remarque 3 Soient $F \in \mathbb{C}\left[\left[S_{\rho}\right]\right]$ un polynôme de degré $\geq 1$ et $w \in \rho$ un vecteur irrationnel, définissant un chemin polygonal $\gamma$ dans les arêtes de $\mathcal{N}_{\rho}(F)$. La démonstration du théorème 2 montre que, associées à chaque arête $e$ de $\gamma$, il existe $l_{e}$ racines de $F$ telles que leur exposant initial par rapport à $w$ est l'inclinaison $q_{e}$.

\subsection{Rapport avec les paramétrisations des singularités quasi-ordinaires}

Théorème 3 Soit $F=\sum_{j=0}^{n} a_{j} Y^{j}$ un polynôme réduit de degré $n \geq 1$ avec $a_{j} \in \mathbb{C}\left\{\left\{S_{\rho}\right\}\right\}$.

1. Pour tout cône $\tau$ de dimension d de l'éventail associé au polyèdre $\mathcal{N}_{\rho}\left(a_{n} \Delta_{Y} F\right)$, il existe $k \in \mathbb{N}$ tel que $F$ se décompose dans l'anneau $\mathbb{C}\left\{\left\{\frac{1}{k} S_{\tau}\right\}\right\}$. 
2. Si $a_{0} \neq 0$, pour tout cône $\tau$ de dimension d de l'éventail associé au polyèdre $\mathcal{N}_{\rho}\left(a_{n} \Delta_{Y} F\right)+$ $Q_{\rho}(F)$ il existe $k \in \mathbb{N}$ tel que $F$ se décompose dans l'anneau $\mathbb{C}\left\{\left\{\frac{1}{k} S_{\tau}\right\}\right\}$, et de plus les racines de F sont des unités.

Preuve Soit $\tau$ un cône de dimension $d$ de l'éventail associé au polyèdre $\mathcal{N}_{\rho}\left(a_{n} \Delta_{Y} F\right)$. Par définition de $\rho$-polyèdre de Newton, le cône $\tau$ est contenu dans $\rho$, et on a l'homomorphisme d'algèbres $\mathbb{C}\left\{S_{\rho}\right\} \hookrightarrow \mathbb{C}\left\{S_{\tau}\right\}$ qui permet de considérer $F$ comme élément de $\mathbb{C}\left\{\left\{S_{\tau}\right\}\right\}[Y]$.

Tout vecteur irrationnel $w \in \tau$ atteint sa valeur minimale sur les exposants de $a_{n}$ au même point $u_{n}$. On montre par récurrence sur $n$ qu'il existe $q_{0} \in \mathbb{Z}^{d}$ tel que le polyèdre $\mathcal{N}_{\tau}(F)$ est contenu dans le cône affine:

$$
W:=\left\{\left(u_{n}, n\right)+\lambda\left(q_{0},-1\right)+\left(u^{\prime}, 0\right) / \lambda \in[0, n], u^{\prime} \in \tau^{\vee}\right\} .
$$

Si $n=1$, il suffit de prendre $q \in \mathbb{Z}^{d}$ tel que $\mathcal{N}_{\tau}\left(a_{0}\right)$ soit contenu dans le cone affine $u_{n}+q+\tau^{\vee}$. Si $n>1$, par récurrence on a construit $q$ pour le polynôme $\left(F-a_{0}\right) Y^{-1}$. Il suffit de prendre $q_{0} \in \mathbb{Z}^{d}$ tel que le polyèdre $u_{n}+n q+\tau^{\vee}+\mathcal{N}_{\tau}\left(a_{0}\right)$ soit contenu dans le cone affine $u_{n}+n q_{0}+\tau^{\vee}$.

Nous notons $p_{0}$ le point $u_{n}+n q_{0}, e_{0}$ le segment $\left[\left(u_{n}, n\right),\left(p_{0}, 0\right)\right]$ et $\pi_{e_{0}}: \mathbb{R}^{d} \times \mathbb{R} \rightarrow$ $\mathbb{R}^{d} \times\{0\}$ la projection parallèlement à l'arête $e_{0}$. On a $\pi_{e_{0}}(u, v)=u+v q_{0}$.

On définit le changement:

$$
G=X^{-p_{0}} F\left(X^{q_{0}} Y\right) .
$$

On en déduit:

1. Si $F=\sum_{j=0}^{n} a_{j} Y^{j}$ avec $a_{i} \in \mathbb{C}\left\{\left\{S_{\tau}\right\}\right\}$ on obtient que $G=\sum_{j=0}^{n} a_{j} X^{j q_{0}-p_{0}} Y^{j}$, et donc l'exposant de $G$ qui correspond à l'exposant $(u, j) \operatorname{de} F$ est $\left(\pi_{e_{0}}(u)-p_{0}, j\right)$. Par construction, comme $\mathcal{N}_{\tau}(F) \subset W$, le vecteur $\pi_{e_{0}}(u)-p_{0}$ appartient au cône $\tau^{\vee}$. Ceci implique que $G$ est un polynôme dans l'anneau $\mathbb{C}\left\{S_{\tau}\right\}$. De plus, l'exposant de $G$ qui correspond à l'exposant $\left(u_{n}, n\right)$ de $F$ est $(0, n)$, donc $G\left(z_{\tau}, Y\right) \in \mathbb{C}[Y]$ est un polynôme de degré $n$.

2. La quasi-homogéneité et l'homogéneité du discriminant générique impliquent que le discriminant de $G$ par rapport à $Y$ est de la forme $\Delta_{Y} G=X^{u_{0}} \varepsilon$ où $\varepsilon$ est une unité dans l'anneau $\mathbb{C}\left\{S_{\tau}\right\}$.

En appliquant le théorème 1 , on voit qu'il existe $k \in \mathbb{N}$ tel que $G$ se décompose dans l'anneau $\mathbb{C}\left\{\frac{1}{k} S_{\tau}\right\}$. Les racines correspondantes de $F$ sont dans $\mathbb{C}\left\{\left\{\frac{1}{k} S_{\tau}\right\}\right\}$.

Soit $\tau$ est un cône de dimension $d$ de la subdivision finie de $\rho$ induite par le polyèdre $\mathcal{N}_{\rho}\left(a_{n} \Delta_{Y} F\right)+Q_{\rho}(F)$; vérifions que les racines construites sont des unités dans $\mathbb{C}\left\{\left\{S_{\tau}\right\}\right\}$.

Soit $w \in \tau$ un vecteur irrationnel. D'après le théorème 2 , il existe un cône rationnel strictement convexe $\sigma$ qui contient $w$, et $k \in \mathbb{N}$ tels que le polynôme $F$ se décompose sur l'anneau $\mathbb{C}\left(\left(\frac{1}{k} S_{\sigma}\right)\right)$.

Puisque le vecteur $w \in \tau \cap \sigma$ est irrationnel, le cône rationnel $\tau \cap \sigma$ est nécessairement un cône de dimension $d$, et son cône dual $\tau^{\vee}+\sigma^{\vee}$ est strictement convexe. L'anneau intègre $\mathbb{C}\left(\left(\frac{1}{k} S_{\tau \cap \sigma}\right)\right)$ contient $\mathbb{C}\left(\left(\frac{1}{k} S_{\sigma}\right)\right)$ et $\mathbb{C}\left(\left(\frac{1}{k} S_{\tau}\right)\right)$ comme sous-anneaux.

D'abord, les racines de $F$ obtenues par le théorème 1 sont dans l'anneau $\mathbb{C}\left\{\left\{\frac{1}{k} S_{\tau}\right\}\right\}$, et donc elles doivent coïncider avec les racines obtenues à la Newton-Puiseux. Nous affirmons que ces racines sont des éléments inversibles dans l'anneau $\mathbb{C}\left\{\left\{\frac{1}{k} S_{\tau}\right\}\right\}$. Par hypothèse, chaque élément irrationnel $w \in \tau$ définit le même chemin $\gamma$ dans les arêtes de 
$\mathcal{N}_{\tau}(F)$. Donc l'exposant initial $u$ par rapport à $w$ d'une racine $\phi$ ne dépend pas de $w \in \tau$ (par la remarque 3 ). On obtient que $\phi=X^{u} \varepsilon$ où $\varepsilon$ est une unité de $\mathbb{C}\left\{\frac{1}{k} S_{\tau}\right\}$, c'est-à-dire que $\phi$ est une unité dans $\mathbb{C}\left\{\left\{\frac{1}{k} S_{\tau}\right\}\right\}$.

On obtient aussi la version polynomiale du théorème précedent:

Corollaire 1 Soit un polynôme $F=\sum a_{j} Y^{j} \in \mathbb{C}\left[X_{1}, \ldots, X_{d}\right][Y]$ réduit de degré $n \geq 1$.

1. Si le polytope $\mathcal{P}=\mathcal{P}\left(a_{n} \Delta_{Y} F\right)$ est de dimension d, pour tout cône $\tau$ de dimension d de l'éventail associé à $\mathcal{P}$ il existe $k \in \mathbb{N}$ tel que $F$ se décompose dans l'anneau $\mathbb{C}\left\{\left\{\frac{1}{k} S_{\tau}\right\}\right\}$.

2. Si $a_{0} \neq 0$ et si $\tau$ est un cône associé à un sommet du polytope $\mathcal{P}\left(\Delta_{Y} F\right)+\mathcal{Q}(F)$, toutes les racines de $F$ sont des unités dans $\mathbb{C}\left\{\left\{\frac{1}{k} S_{\tau}\right\}\right\}$.

Preuve Si le polytope $\mathcal{P}\left(a_{n} \Delta_{Y} F\right)$ est de dimension $d$, le cône $\tau$ associé à un sommet de $\mathcal{P}\left(a_{n} \Delta_{Y} F\right)$ est de dimension $d$. On applique le théorème 3 à $F$ vu comme élément de $\mathbb{C}\left\{\left\{S_{\tau}\right\}\right\}[Y]$.

Si le polytope $\mathcal{P}:=\mathcal{P}\left(\Delta_{Y} F\right)+\mathcal{Q}(F)$ est de dimension $<d$, le cône $\tau$ associé à un sommet de $\mathcal{P}$ est rationnel de dimension $d$ mais il n'est pas strictement convexe. Le cône $\tau^{\vee}$ est strictement convexe et $\tau$ définit l'algèbre $\mathbb{C}\left[\left[S_{\tau}\right]\right]$. Si $\sigma \subset \tau$ est un cône strictement convexe de dimension $d$, on considére $F$ comme élément de $\mathbb{C}\left\{\left\{S_{\sigma}\right\}\right\}[Y]$ et on obtient que il existe $k \in \mathbb{N}$ tel que $F$ se décompose dans l'anneau $\mathbb{C}\left\{\left\{\frac{1}{k} S_{\sigma}\right\}\right\}$.

On peut recouvrir le cône $\tau$ par un nombre fini de cônes rationnels strictement convexes de dimension d, $\left\{\sigma_{i}\right\}_{1 \geq i \geq s}$, tels que $\sigma_{i} \cap \sigma_{i+1}$ soit d'intérieur non vide, pour $i=1, \ldots, s-1$. Ceci implique que les racines de $F$ obtenues par le théorème 1 correspondant à $\sigma_{i}$ et à $\sigma_{i+1}$ vont coïncider, et le terme initial d'une racine par rapport à la valuation induite par un vecteur irrationel $w$ ne dépend pas de $w \in \sigma_{i} \cup \sigma_{i+1}$. Donc toutes les racines de $F$ sont des séries à exposants dans un translaté du cône $\bigcap_{i=1}^{s} \sigma_{i}^{\vee}=\tau^{\vee}$.

Remarque 4 Soit $F \in \mathbb{C}\left[X_{1}, \ldots, X_{d}\right][Y]$ un polynôme de degré $n$ tel que 0 soit une racine simple de $F(0, Y)$, le théorème des fonctions implicites garantit qu'il existe une unique série $\phi \in \mathbb{C}\left\{X_{1}, \ldots, X_{d}\right\}$ telle que $F(\phi)=0$. Si on a $a_{n} \Delta_{Y} F=X^{u} \varepsilon$ où $\varepsilon(0) \neq 0$ le théorème 3 montre que les exposants de $\phi$ sont dans un translaté entier du cône dual associé au sommet $u$ de $\mathcal{P}\left(a_{n} \Delta_{Y} F\right)$ lorsque ce polytope est de dimension $d$. (Voir l'exemple 1).

\section{Application aux polyèdres de Newton du discriminant et du résultant}

\subsection{Les conditions discriminantales pour le polyèdre de Newton}

Suivant [9], on dit que un polynôme $F \in \mathbb{C}\left(\left(S_{\rho}\right)\right)[Y]$ vérifie la condition discriminantale si pour toute arête admissible $e$ de son $\rho$-polyèdre de Newton, le polynôme $f_{e}$ n'a que des racines simples.

Théorème 4 Soit $F=\sum_{k=0}^{n} a_{k} Y^{k}$ un polynôme à coefficients dans $\mathbb{C}\left(\left(S_{\rho}\right)\right)$ tels que $a_{0} a_{n} \neq$ 0 . On a l'inclusion de polyèdres

$$
\mathcal{N}_{\rho}\left(a_{0}\right)+\mathcal{N}_{\rho}\left(a_{n}\right)+\mathcal{N}_{\rho}\left(\Delta_{Y} F\right) \subseteq Q_{\rho}(F)
$$


où $\Delta_{Y} F$ est le discriminant de $F$ par rapport à $Y$. On a l'égalité si $F$ vérifie la condition discriminantale.

Preuve On va trouver les conditions génériques que doivent vérifier les coefficients des termes qui apparaissent dans $F$, pour garantir l'égalité dans le théorème.

Soit $w \in \rho$ un vecteur irrationnel, et soit $\gamma$ le chemin monotone défini par $w$ dans les arêtes du $\rho$-polyèdre de Newton. Le chemin $\gamma$ a des sommets $\left\{p_{0}, p_{i_{1}}, \ldots, p_{i_{t}}, p_{n}\right\}$ dans $\mathcal{N}_{\rho}(F)$, avec $p_{j}=\left(u_{j}, j\right)$ pour $j \in\left\{0=i_{0}, i_{1}, \ldots, i_{t}, i_{t+1}=n\right\}$ avec $i_{0}<\cdots<i_{t+1}$. Nous notons $q_{r}:=\frac{-u_{i_{r}}+u_{i_{r}-1}}{i_{r}-i_{r-1}} \in \mathbb{O}^{d}$ et $l_{r}:=i_{r}-i_{r-1}$ l'inclinaison et la longueur du segment $e_{r}=\left[p_{i_{r-1}}, p_{i_{r}}\right]$ du chemin $\gamma$, pour $r=1, \ldots, t+1$.

D'après le théorème 2 , il existe un cône rationnel strictement convexe $\sigma_{w}$, et $k \in \mathbb{N}$ tels que $F$ se décompose dans l'anneau $\mathbb{C}\left(\left(\frac{1}{k} S_{\sigma_{w}}\right)\right)[Y]$. À chaque segment $e_{r}=\left[p_{i_{r-1}}, p_{i_{r}}\right] \mathrm{du}$ chemin $\gamma$ sont associées $i_{r}-i_{r-1}$ racines de $F$ de la forme:

$$
\phi_{j}=c_{j} X^{q_{r}}+\cdots,
$$

où $q_{r}$ est l'inclinaison du segment $e_{r}$, et $c_{j}$ parcourt les racines de $f_{e_{r}}$ comptées avec leur multiplicité. De plus $q_{r}$ est l'exposant initial par rapport à $w$ des termes qui apparaissent dans $\phi_{j}$. On indexe les racines $\phi_{j}$ correspondant à $e_{r}$, par $j \in \mathcal{A}_{r}:=\left\{i_{r-1}+1, \ldots, i_{r}\right\}$.

En appliquant le lemme 5, on voit que parmi les termes qui peuvent apparaître dans $\phi_{k}-\phi_{j}$, celui d'exposant de le plus petit par rapport à $<_{w}$ est égal à:

$$
\begin{cases}\left(c_{k}-c_{j}\right) X^{q_{r}} & \text { si } k, j \in \mathcal{A}_{r} \\ c_{j} X^{q_{m}} & \text { si } k \in \mathcal{A}_{r}, j \in \mathcal{A}_{m} \text { et } r<m .\end{cases}
$$

Comme,

$$
\Delta_{Y} F=(-1)^{\frac{1}{2} n(n-1)} a_{n}^{2(n-1)} \prod_{k<j}\left(\phi_{k}-\phi_{j}\right)^{2},
$$

le terme d'exposant le plus petit par rapport à $<_{w}$ qui peut apparaître dans $a_{0} a_{n} \Delta_{Y} F$ est égal a $A B C$ où:

$$
\begin{gathered}
A=(-1)^{\frac{1}{2} n(n-1)} \alpha_{p_{0}} \alpha_{p_{n}}^{2 n-1} X^{u_{0}+(2 n-1) u_{n}} \\
B=\prod_{r=1}^{t+1} \prod_{i_{r-1}<k_{r}<j_{r} \leq i_{r}}\left(c_{k_{r}}-c_{j_{r}}\right)^{2} X^{2 q_{r}} \\
C=\prod_{r=1}^{t} \prod_{k_{r} \in \mathcal{A}_{r}} \prod_{m=r+1}^{t+1} \prod_{j_{m} \in \mathcal{A}_{m}} c_{j_{m}}^{2} X^{2 q_{m}}
\end{gathered}
$$

L'exposant correspondant à $B$ est:

$$
\begin{aligned}
& 2\left(\left(\begin{array}{c}
i_{1} \\
2
\end{array}\right) q_{1}+\left(\begin{array}{c}
i_{2}-i_{1} \\
2
\end{array}\right) q_{2}+\cdots+\left(\begin{array}{c}
i_{t+1}-i_{t} \\
2
\end{array}\right) q_{t+1}\right) \\
& \quad=\left(i_{1}-1\right)\left(u_{0}-u_{i_{1}}\right)+\left(i_{2}-i_{1}-1\right)\left(u_{i_{1}}-u_{i_{2}}\right)+\cdots+\left(i_{t+1}-i_{t}-1\right)\left(u_{i_{t}}-u_{i_{t+1}}\right)
\end{aligned}
$$




$$
\begin{gathered}
=\left(i_{1}-1\right) u_{0}+\left(i_{2}-2 i_{1}\right) u_{i_{1}}+\left(i_{3}-2 i_{2}+i_{1}\right) u_{i_{2}} \\
+\cdots+\left(n-2 i_{t}+i_{t-1}\right) u_{i_{t}}+\left(-n+i_{t-1}\right) u_{n}
\end{gathered}
$$

L'exposant correspondant à $C$ est:

$$
\begin{aligned}
& 2\left(\sum_{r=1}^{t}\left(i_{r}-i_{r-1}\right) \sum_{m=r+1}^{t+1} q_{m}\left(i_{m}-i_{m-1}\right)\right) \\
& \quad=2\left(\sum_{r=1}^{t}\left(i_{r}-i_{r-1}\right)\left(-u_{n}+u_{i_{r}}\right)\right) \\
& \quad=2\left(i_{1} u_{i_{1}}+\left(i_{2}-i_{1}\right) u_{i_{2}}+\cdots+\left(i_{t}-i_{t-1}\right) u_{i_{t}}-i_{t} u_{n}\right) .
\end{aligned}
$$

L'exposant $u$ correspondant à $A B C$ coïncide avec $2 \int \gamma$, (voir la formule (1), Section 3.3). Clairement, le coefficient correspondant à $A B C$ est non nul si et seulement si les segments du chemin $\gamma$ vérifient la condition discriminantale. Ceci termine la preuve, parce que le vecteur irrationnel $w$ est arbitraire.

Corollaire 2 Avec les notations précedentes, le coefficient du terme de la série $a_{0} a_{n} \Delta_{Y} F$ d'exposant égal à $2 \int \gamma$ est:

$$
c(\gamma):=(-1)^{k} \alpha_{p_{i_{0}}} \alpha_{p_{i_{1}}}^{2} \cdots \alpha_{p_{i_{t}}}^{2} \alpha_{p_{i_{t+1}}} \Delta f_{e_{1}} \cdots \Delta f_{e_{t+1}}
$$

ò̀ $f_{e_{r}}=\alpha_{p_{i_{r-1}}}+\cdots+\alpha_{p_{i_{r}}} t^{l_{r}}$ est le polynôme de l'arête $e_{r}=\left[p_{i_{r-1}}, p_{i_{r}}\right] d u$ chemin $\gamma$, son discriminant est $\Delta f_{e_{r}}$ et $k=\frac{1}{2}\left(n(n-1)+\sum_{r=1}^{t+1} l_{r}\left(l_{r}-1\right)\right)$.

Preuve En utilisant que $\Delta f_{e_{r}}=(-1)^{\frac{1}{2} l_{r}\left(l_{r}-1\right)} \alpha_{p_{i_{r}}^{2\left(l_{r}-1\right)}}^{\prod_{i_{r-1}<k_{r}<j_{r} \leq i_{r}}}\left(c_{k_{r}}-c_{j_{r}}\right)^{2}$ on obtient que le coefficient de $B$ est

$$
\prod_{r=1}^{t+1}(-1)^{\frac{1}{2} l_{r}\left(l_{r}-1\right)} \alpha_{p_{i_{r}}}^{-2\left(l_{r}-1\right)} \Delta f_{e_{r}}
$$

Comme le produit des racines de $f_{e_{m}}$ est égal à $(-1)^{l_{m}} \frac{\alpha_{p_{i_{m-1}}}}{\alpha_{p_{i_{m}}}}$, on déduit que le le coefficient de $C$ est

$$
\prod_{r=1}^{t} \prod_{k_{r} \in \mathcal{A}_{r}} \prod_{m=r+1}^{t+1}\left(\frac{\alpha_{p_{i_{m-1}}}}{\alpha_{p_{i_{m}}}}\right)^{2}=\prod_{r=1}^{t} \prod_{k_{r} \in \mathcal{A}_{r}}\left(\frac{\alpha_{p_{i_{r}}}}{\alpha_{p_{i_{t+1}}}}\right)^{2}=\prod_{r=1}^{t}\left(\frac{\alpha_{p_{i_{r}}}}{\alpha_{p_{i_{t+1}}}}\right)^{2 l_{r}} .
$$

Donc le coefficient de $A B C$ est:

$$
(-1)^{k} \Delta f_{e_{1}} \cdots \Delta f_{e_{t+1}} \alpha_{p_{i_{0}}} \alpha_{p_{i_{t+1}}}^{2 n-1-2\left(n-l_{t+1}\right)-2\left(l_{t+1}-1\right)} \prod_{r=1}^{t} \alpha_{p_{i_{r}}}^{2 l_{r}-2\left(l_{r}-1\right)}=c(\gamma)
$$

On déduit des théorèmes 3 et 4 : 
Corollaire 3 Soit le polynôme $F=\sum_{k=0}^{n} a_{k} Y^{k}$, où $a_{k}$ sont des séries dans $\mathbb{C}\left(\left(S_{\rho}\right)\right)$ telles que $a_{0} a_{n} \neq 0$. Si $F$ vérifie la condition discriminantale, pour tout cône $\tau$ de dimension d de l'éventail associé au polyèdre $Q_{\rho}(F)$, il existe $k \in \mathbb{N}$ tel que $F$ se décompose dans l'anneau $\mathbb{C}\left(\left(\frac{1}{k} S_{\tau}\right)\right)$, et de plus les racines de $F$ sont des unités.

Remarque 5 Le corollaire 4.1 de [9] énonce une "version polynomiale" incorrecte du corollaire précedent. Il est dit que des racines de $F$ correspondants aux cônes associés aux sommets différents du polytope-fibre sont différents. Supposons que le polytope $\mathcal{P}:=$ $\mathcal{P}\left(\Delta_{Y} F\right)+\mathcal{P}\left(a_{n}\right)$ soit de dimension $d$, et que l'éventail $\Sigma$ associé au polytope-fibre soit une sous-division stricte de l'éventail $\Sigma^{\prime}$ associé à $\mathcal{P}$. Par le corollaire 1 , les racines de $F$ correspondants aux cônes de $\Sigma$ qui subdivisent un cône $\tau \in \Sigma^{\prime}$ de dimension $d$ vont coïncider dans l'anneau $\mathbb{C}\left\{\left\{\frac{1}{k} S_{\tau}\right\}\right\}$. Elles ne seront pas toutes des unités dans cet anneau. (Voir l'exemple 1).

Corollaire 4 Soit le polynôme $F=\sum_{i=1}^{n} a_{i} Y^{i}$ où $a_{i}$ sont des polynômes dans $\mathbb{C}\left[X_{1}, \ldots, X_{d}\right]$ tels que $a_{0} a_{n} \neq 0$. On a l'inclusion de polytopes

$$
\mathcal{P}\left(a_{0}\right)+\mathcal{P}\left(a_{n}\right)+\mathcal{P}\left(\Delta_{Y} F\right) \subseteq \mathcal{Q}(F)
$$

et on a l'égalité si et seulement si le polynôme F vérifie la condition discriminantale.

Preuve Soit $\rho$ un cône de dimension $d$ strictement convexe. On va considérer le polynôme $F$ comme un élément de l'anneau $\mathbb{C}\left[\left[S_{\rho}\right]\right][Y]$. En appliquant le théorème 4 pour chaque $w \in \rho$, on voit que $\mathcal{Q}(F)+\rho^{\vee} \supseteq \mathcal{P}\left(a_{0}\right)+\mathcal{P}\left(a_{n}\right)+\mathcal{P}\left(\Delta_{Y} F\right)+\rho^{\vee}$, et que l'on a l'égalité si et seulement si toutes les arêtes admissibles du polyèdre $\mathcal{P}(F)+\rho^{\vee} \times\{0\}$ vérifient la condition discriminantale. Ceci termine la preuve parce que $\rho$ est arbitraire.

Remarque 6 En utilisant le corollaire 4 et le théorème 7.3 de [3], on peut déduire de ce qui précède les théorèmes 2.2 et 2.3 , Chap. 12, de [6]. Ces résultats donnent le polytope de Newton du discriminant générique (c'est-à-dire le discriminant du polynôme $F=X_{n} Y^{n}+$ $\cdots+X_{1} Y+X_{0} \in \mathbb{C}\left[X_{0}, X_{1}, \ldots, X_{n}\right][Y]$ par rapport à $\left.Y\right)$ et les coefficients des termes correspondant aux sommets du polytope.

En effet, le polytope $\mathcal{P}(F)$ est un simplexe de dimension $n$, de sommets $\left(u_{j}, j\right) \in \mathbb{R}^{n+1} \times$ $\mathbb{R}$, où $\left\{u_{j}\right\}_{j=0}^{n}$ sont les vecteurs de la base canonique dans $\mathbb{R}^{n+1}$. Comme le polynôme $F$ vérifie la condition discriminantale on a $Q(F)=\mathcal{P}\left(X_{0} X_{n} \Delta_{Y} F\right)$. Chaque sous-ensemble $\left\{i_{1}, \ldots, i_{s}\right\}$ de $\{1, \ldots, n-1\}$ correspond de manière unique à un chemin monotone $\gamma_{\left\{i_{1}, \ldots, i_{s}\right\}}$ dans les arêtes de $\mathcal{P}(F)$. Comme $\mathcal{P}(F)$ est un simplexe, il existe un vecteur irrationnel $w \in\left(\mathbb{R}^{n+1}\right)^{*}$ définissant le chemin $\gamma_{\left\{i_{1}, \ldots, i_{s}\right\}}$. Le sommet de l'intégrale de Minkowski $\int \gamma_{\left\{i_{1}, \ldots, i_{s}\right\}}$ est décrit par la formule (1). En appliquant le corollaire 2, on obtient aussi le coefficient correspondants aux sommets du polytope de Newton du discriminant générique. 


\subsection{Application au polyèdre de Newton du résultant}

On dira que deux polynômes $F, G \in \mathbb{C}\left(\left(S_{\rho}\right)\right)[Y]$ vérifient la condition résultante si pour toute paire d'arêtes $e$ de $\mathcal{N}_{\rho}(F)$ et $e^{\prime} \operatorname{de} \mathcal{N}_{\rho}(G)$ ayant la même inclinaison, les polynômes des arêtes respectives $f_{e}, g_{e}^{\prime} \in \mathbb{C}[t]$ n'ont pas de racines en commun.

Soit $w$ un vecteur irrationnel dans un cône $\tau$ de dimension $d$ de l'éventail associé au polyèdre $Q_{\rho}(F)+Q_{\rho}(G)$. Le vecteur $w$ détermine des chemins monotones uniques $\gamma_{F}$ et $\gamma_{G}$ dans les arêtes des $\rho$-polyèdres de Newton de $F$ et de $G$. Le chemin $\gamma_{F}$ a des arêtes $e_{i}$ d'inclinaisons $q_{i}$, pour $i=1, \ldots, m$ et par le lemme 5 on a $q_{m}<_{w} \cdots<_{w} q_{1}$. Le chemin $\gamma_{G}$ a des arêtes $e_{j}^{\prime}$ d'inclinaisons $q_{j}^{\prime}$, pour $j=1, \ldots, m^{\prime}$ tels que $q_{m^{\prime}}^{\prime}<_{w} \cdots<_{w} q_{1}^{\prime}$.

Par contre, l'ordre défini par $w$ dans $q_{1}, \ldots, q_{m}, q_{1}^{\prime}, \ldots, q_{m^{\prime}}^{\prime}$ peut varier lorsque $w$ parcourt $\tau$. Nous considérons la subdivision finie rationnelle la moins fine de $\tau$ possédant la propriété suivante: des vecteurs irrationnels qui sont dans le même cône de la subdivision définissent le même ordre sur l'ensemble des inclinaisons $q_{1}, \ldots, q_{m}, q_{1}^{\prime}, \ldots, q_{m^{\prime}}^{\prime}$. On définit de cette manière une subdivision $\Sigma$ de l'éventail associé à $Q_{\rho}(F)+\mathcal{Q}_{\rho}(G)$.

Proposition 1 L'éventail associé à l'intégrale de Minkowski $Q_{\rho}(F G)$ est égal à $\Sigma$.

Preuve Soit $\gamma_{F G}$ le chemin monotone dans le polyèdre $\mathcal{N}_{\rho}(F G)=\mathcal{N}_{\rho}(F)+\mathcal{N}_{\rho}(G)$ défini par un vecteur irrationnel $w \in \tau \in \Sigma$. Chaque point $\gamma(t)$ est la somme de deux points situés dans les chemins $\gamma_{F}$ et $\gamma_{G}$ définis par $w$ dans les polyèdres respectifs. Clairement on a $\gamma_{F G}\left(n+n^{\prime}\right)=\gamma_{F}(n)+\gamma_{G}\left(n^{\prime}\right)$. Si $q_{m} \geq_{w} q_{m}^{\prime}$, le segment $l_{m+m^{\prime}}:=\gamma_{G}\left(n^{\prime}\right)+e_{m}$ est contenu dans $\gamma_{F G}$. Ce segment n'est pas une arête de $\gamma_{F G}$ si et seulement si, on a $q_{m}=q_{m}^{\prime}$. Par récurrence, on subdivise $\gamma_{F G}$ en $m+m^{\prime}$ segments, $l_{1}, \ldots, l_{m+m^{\prime}}$, tels qu'il existe une bijection $\left\{l_{1}, \ldots, l_{m+m^{\prime}}\right\} \rightarrow\left\{e_{1}, \ldots, e_{m}, e_{1}^{\prime}, \ldots, e_{m^{\prime}}^{\prime}\right\}$ qui préserve l'inclinaison et la longueur. De plus, $\gamma_{F G}$ est complètement déterminé par $\gamma_{F}, \gamma_{G}$ et l'ordre des inclinaisons. Ceci implique que $\Sigma$ est un éventail plus fin que l'éventail associé à $Q_{\rho}(F G)$.

Réciproquement, si $w, w^{\prime}$ sont des vecteurs irrationnels dans un cône de l'éventail associé au polyèdre $Q_{\rho}(F G)$, ils définissent un unique chemin monotone $\gamma$ et par le lemme 5 les inclinaisons de ses arêtes ont le même ordre par rapport à $<_{w}$ et $<_{w^{\prime}}$, donc $w$, $w^{\prime}$ sont dans le même cône de $\Sigma$.

Proposition 2 Soient $F, G \in \mathbb{C}\left(\left(S_{\rho}\right)\right)[Y]$ des polynômes de degrés $n, n^{\prime} \geq 1$ ayant des termes constants non nuls. Si F, G vérifient la condition résultante, alors:

1. L'éventail $\Sigma$ associé au polyèdre-fibre $Q_{\rho}(F G)$ est une subdivision de l'éventail du $\rho$ polyèdre de Newton du résultant de $F$ et $G$.

2. Soit $\tau \in \Sigma$ un cône de dimension $d$, définissant les chemins monotones $\gamma_{F}$, $\gamma_{G}$ et $\gamma_{F G}$ dans les polyèdres de Newton $\mathcal{N}_{\rho}(F), \mathcal{N}_{\rho}(G)$ et $\mathcal{N}_{\rho}(F G)$. Le sommet du $\rho$-polyèdre de Newton du résultant de F et $G$ associé a $\tau$ est $\int \gamma_{F G}-\int \gamma_{F}-\int \gamma_{G}$.

Preuve Soit $w \in \tau \in \Sigma$ un vecteur irrationnel, on montre d'abord que l'exposant le plus petit par rapport à $<_{w}$ qui peut apparaître dans $\operatorname{Res}(F, G)$ est le même pour tout $w \in \tau$.

Le chemin $\gamma_{F}$ a des arêtes $e_{r}=\left[p_{r-1}, p_{r}\right]$ de pente $q_{r}$ est de longueur $l_{r}$ pour $r=$ $1, \ldots, m$. Le polynôme associé à l'arête $e_{r}$ est $f_{r}=\alpha_{p_{r-1}}+\cdots+\alpha_{p_{r}} t^{l_{r}}$. Nous notons $\left\{c_{r}^{1}, \ldots, c_{r}^{l_{r}}\right\}$ ses racines comptées avec multiplicité. 
Le chemin $\gamma_{G}$ a des arêtes $e_{s}^{\prime}=\left[p_{s-1}^{\prime}, p_{s}^{\prime}\right]$ de pente $q_{s}^{\prime}$ est de longueur $l_{s}^{\prime}$ pour $s=$ $1, \ldots, m^{\prime}$. Le polynôme associé à l'arête $e_{s}^{\prime}$ est $g_{s}=\beta_{p_{s-1}^{\prime}}+\cdots+\beta_{p_{s}^{\prime}} t_{s}^{\prime}$. Nous notons $\left\{d_{s}^{1}, \ldots, d_{s}^{l_{s}^{\prime}}\right\}$ ses racines comptées avec multiplicité.

Par le théorème 2, le terme initial par rapport à $<_{w}$, d'une racine $\phi_{r}^{i}$ de $F$ correspondant au segment $e_{r}$ est $c_{r}^{i} X^{q_{r}}$, et celui d'un racine $\psi_{s}^{j}$ de $G$ correspondant au segment $e_{s}^{\prime}$ est $d_{s}^{j} X^{q_{s}^{\prime}}$.

Si $a_{n}$ et $b_{n^{\prime}}$ sont les coefficients des termes de degré $n$ et $n^{\prime}$ de $F$ et $G$ respectivement, on a $\operatorname{Res}(F, G)=a_{n}^{n^{\prime}} b_{n^{\prime}}^{n} \prod\left(\phi_{r}^{i}-\psi_{s}^{j}\right)$. Le coefficient du terme d'exposant le plus petit par rapport à $<_{w}$ qui peut apparaître dans $\operatorname{Res}(F, G)$ est le produit $A B C D$ où le facteur $A$ correspond à $a_{n}^{n^{\prime}} b_{n^{\prime}}^{n}$ :

$$
\begin{gathered}
A=\alpha_{p_{m}}^{n^{\prime}} \beta_{p_{m}^{\prime}}^{n}, \\
B=\prod_{q_{r}=q_{s}^{\prime}}^{r, s} \prod_{i=1, \ldots, l_{r}} \prod_{j=1, \ldots, l_{s}^{\prime}}\left(c_{r}^{i}-d_{s}^{j}\right), \\
C=\prod_{q_{r}>w q_{s}^{\prime}} \prod_{j=1, \ldots, l_{s}^{\prime}} \prod_{i=1, \ldots, l_{r}} c_{r}^{i}, \\
D=\prod_{q_{r}<w}^{r, s} \prod_{j} \prod_{j=1, \ldots, l_{s}^{\prime}} \prod_{i=1, \ldots, l_{r}}-d_{s}^{j} .
\end{gathered}
$$

Comme $F$ et $G$ vérifient la condition résultante, on a $A B C D \neq 0$, et le terme obtenu ne varie pas lorsque $w$ parcourt $\tau$.

Nous notons $u_{\Delta(F)}, u_{D(G)}$ et $u_{\Delta(F G)}$ l'exposant le plus petit par rapport à $<_{w}$ qui peut apparaître parmi les exposants du discriminant de $F, G$ et $F G$ respectivement. Par le théorème 4 on a:

$$
\begin{gathered}
2 \int \gamma_{F}=\gamma_{F}(0)+\gamma_{F}(n)+u_{\Delta(F)} \\
2 \int \gamma_{G}=\gamma_{G}(0)+\gamma_{G}\left(n^{\prime}\right)+u_{\Delta(G)} \\
2 \int \gamma_{F G}=\gamma_{F G}(0)+\gamma_{F G}\left(n+n^{\prime}\right)+u_{\Delta(F G)} .
\end{gathered}
$$

On considère l'expression $(\operatorname{Res}(F, G))^{2} \Delta_{Y}(F) \Delta_{Y}(G)=\Delta_{Y}(F G)$ en fonction des racines de $F$ et de $G$ et on déduit que si $u_{0}$ est l'exposant initial par rapport à $<_{w} \operatorname{de} \operatorname{Res}(F, G)$ :

$$
2 u_{0}=u_{\Delta(F G)}-u_{\Delta(F)}-u_{\Delta(G)}=2 \int \gamma_{F G}-2 \int \gamma_{F}-2 \int \gamma_{G},
$$

parce que l'on a $\gamma_{F G}(0)=\gamma_{F}(0)+\gamma_{G}(0)$ et $\gamma_{F G}\left(n+n^{\prime}\right)=\gamma_{F}(n)+\gamma_{G}\left(n^{\prime}\right)$.

Corollaire 5 Dans les hypothèses de la proposition 2, chaque cône $\tau \in \Sigma$ de dimension d définit un sommet du $\rho$-polyèdre de Newton du résultant de F et $G$ de coefficient:

$$
\alpha_{p_{m}}^{n^{\prime}} \beta_{p_{m}^{\prime}}^{n}\left(\prod_{q_{r}=q_{s}^{\prime}}^{r, s} \alpha_{p_{r}}^{-l_{s}^{\prime}} \beta_{p_{s}^{\prime}}^{-l_{r}} \operatorname{Res}\left(f_{r}, g_{s}\right)\right)\left(\prod_{q_{r}>q_{s}^{\prime}}^{r, s}(-1)^{l_{r} l_{s}^{\prime}}\left(\frac{\alpha_{p_{r-1}}}{\alpha_{p_{r}}}\right)^{l_{s}^{\prime}}\right)\left(\prod_{q_{r}<w q_{s}^{\prime}}^{r, s}\left(\frac{\beta_{p_{s-1}^{\prime}}}{\beta_{p_{s}^{\prime}}}\right)^{l_{r}}\right) \text {. }
$$


Preuve Comme $\operatorname{Res}\left(f_{r}, g_{s}\right)=\alpha_{p_{r}}^{l_{s}^{\prime}} \beta_{p_{s}^{\prime}}^{l_{r}} \prod_{i, j}\left(c_{r}^{i}-d_{s}^{j}\right)$ on a:

$$
B=\prod_{q_{r}=q_{s}^{\prime}}^{r, s} \alpha_{p_{r}}^{-l_{s}^{\prime}} \beta_{p_{s}^{\prime}}^{-l_{r}} \operatorname{Res}\left(f_{r}, g_{s}\right)
$$

En utilisant que $\prod_{i=1, \ldots, l_{r}} c_{r}^{i}=(-1)^{l_{r}} \frac{\alpha_{p_{r-1}}}{\alpha_{p_{r}}}$, et que $\prod_{j=1, \ldots, l_{s}^{\prime}}-d_{s}^{j}=\frac{\beta_{p_{s-1}^{\prime}}}{\beta_{p_{s}^{\prime}}}$ on déduit:

$$
C=\prod_{q_{r}>w q_{s}^{\prime}}^{r, s}(-1)^{l_{r} l_{s}^{\prime}}\left(\frac{\alpha_{p_{r-1}}}{\alpha_{p_{r}}}\right)^{l_{s}^{\prime}}, \quad D=\prod_{q_{r}<w q_{s}^{\prime}}^{r, s}\left(\frac{\beta_{p_{s-1}^{\prime}}}{\beta_{p_{s}^{\prime}}}\right)^{l_{r}} .
$$

Exemple 1 Considérons le polynôme $F=U^{4} V^{2} Y^{5}+U^{3} V^{2} Y^{2}-Y+U^{2} V+V^{2}$. Le discriminant du polynôme $F$ par rapport à $Y$ est

$$
\begin{aligned}
\Delta_{Y} F= & 108 U^{25} V^{15}+3125 U^{24} V^{12}+108 U^{23} V^{16}+12500 U^{22} V^{13} \\
& -2250 U^{22} V^{12}+18750 U^{20} V^{14}-4500 U^{20} V^{13}-27 U^{20} V^{12} \\
& +12500 U^{18} V^{15}-2250 U^{18} V^{14}+1600 U^{17} V^{9}+3125 U^{16} V^{16} \\
& +1600 U^{15} V^{10}-256 U^{12} V^{6} \\
= & U^{12} V^{6} \varepsilon
\end{aligned}
$$

où $\varepsilon$ est une unité de l'anneau $\mathbb{C}\left\{S_{\Delta}\right\}$, où $\Delta=\operatorname{pos}\{(-2,5),(2,-1)\}$. Nous allons montrer de deux manières qu'il existe $k \in \mathbb{N}$ tel que $F$ se décompose dans l'anneau $\mathbb{C}\left\{\left\{\frac{1}{k} S_{\Delta}\right\}\right\}$.

1. D'abord, on définit $G:=U^{6} V^{8} F\left(U^{-2} V^{-2}\right)=Y^{5}+U^{5} V^{6} Y^{2}-U^{4} V^{6} Y+U^{8} V^{9}+U^{6} V^{10}$ et on vérifie que $G \in \mathbb{C}\left\{S_{\Delta}\right\}[Y]$ et que $G\left(z_{\Delta}, Y\right)=Y^{5}$. Comme le discriminant de $F$ est une unité dans $\mathbb{C}\left\{\left\{S_{\Delta}\right\}\right\}$ par le théorème 1 , il existe $k \in \mathbb{N}$ tel que $G$ se décompose dans $\mathbb{C}\left\{\frac{1}{k} S_{\Delta}\right\}$ et donc $F$ se décompose dans $\mathbb{C}\left\{\left\{\frac{1}{k} S_{\Delta}\right\}\right\}$.

2. Le polynôme $F$ vérifie la condition discriminantale donc on a $\mathcal{N}_{\Delta}\left(U^{4} V^{2}\left(U^{2} V+V^{2}\right) \Delta_{Y} F\right)=\mathcal{Q}_{\Delta}(F)$. Le $\Delta$-polyèdre-fibre $\mathcal{Q}_{\Delta}(F)$ a deux sommets correspondant aux chemins monotones cohérents $\left(\gamma_{i}\right)_{i=1,2}$. Le premier, $\gamma_{1}$, correspondant aux termes $U^{4} V^{2} Y^{5}, Y, V^{2}$ et définissant le sommet $2 \int \gamma_{1}=(8,10)$ du polyèdre $Q_{\Delta}(F)$. Le deuxième $\gamma_{2}$, correspondant à $U^{4} V^{2} Y^{5}, Y, U^{2} V$ et définissant le sommet $2 \int \gamma_{2}=(8,9)$. L'éventail associé est la subdivision de $\Delta$ par les cônes $\sigma_{1}=\operatorname{pos}\{(2,-1),(1,2)\}$, et $\sigma_{2}=$ $\operatorname{pos}\{(-2,5),(1,2)\}$. Fixons un vecteur irrationnel $w \in \Delta$. Si $w \in \sigma_{1}$, (resp. $\left.w \in \sigma_{2}\right)$, il détermine le chemin $\gamma_{1}$, (resp. $\left.\gamma_{2}\right)$.

Les 4 racines de $F$ correspondant au segment $e=[(4,2,5),(0,0,1)]$ de $\gamma_{1}$ (resp. de $\left.\gamma_{2}\right)$ par le théorème 2 ont un terme d'exposant $\left(-1,-\frac{1}{2}\right)$. On définit $F_{2}=F\left(Y+\lambda U^{-1} V^{-1 / 2}\right)$ 


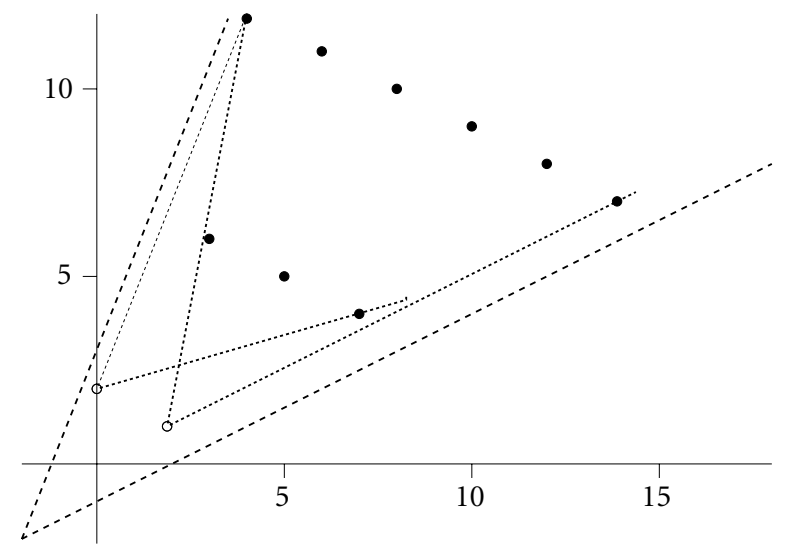

Figure 1: Les sommets noirs sont les exposants de $F_{3}^{(1)}(U, V, 0)$

où $\lambda^{4}=1$.

$$
\begin{aligned}
F_{2}:= & Y^{5} U^{4} V^{2}+5 \lambda Y^{4} U^{3} V^{3 / 2}+10 \lambda^{2} Y^{3} U^{2} V+Y^{2} U^{3} V^{2} \\
& +10 \lambda^{3} Y^{2} U V^{1 / 2}+2 \lambda Y U^{2} V^{3 / 2}+5 Y-Y \\
& +U^{2} V+\lambda^{2} U V+\lambda U^{-1} V^{-1 / 2}-\lambda U^{-1} V^{-1 / 2}+V^{2} .
\end{aligned}
$$

Par la preuve du théorème 2 les exposants des racines correspondant à $e$ sont dans le cône affine de sommet $\pi_{e}(e)=\left(-1, \frac{-1}{2}\right)$ qui contient les exposants de $\pi_{e}\left(\mathcal{N}_{\Delta}\left(F_{2}\right)\right)$ cette-à-dire le cône $\left(-1, \frac{-1}{2}\right)+\operatorname{pos}\{(2,3),(2,1)\}$. Comme le cône pos $\{(2,3),(2,1)\}$ est contenu dans $\Delta^{\vee}$, ces racines sont des éléments de $\mathbb{C}\left\{\left\{\frac{1}{2} S_{\Delta}\right\}\right\}$.

Par ailleurs, $F(0, Y)=-Y$, et en appliquant le théorème des fonctions implicites, il existe un unique $\phi \in \mathbb{C}\{U, V\}$ tel que $F(\phi)=0$. Clairement, $\phi$ doit coïncider avec la série correspondant au segment $[(0,0,1),(0,2,0)]$ déterminé par $w \in \sigma_{1}$ (resp. au segment $[(0,0,1),(0,1,2)]$ déterminé par $\left.w \in \sigma_{2}\right)$.

Pour $w \in \sigma_{1}$, on définit $F_{2}^{(1)}:=F\left(Y+V^{2}\right)$ et on remarque que le terme initial par la valuation $w$ de $F_{2}^{(1)}(0)=U^{4} V^{12}+U^{3} V^{6}+U^{2} V$ est $U^{2} V$. On défini $F_{3}^{(1)}:=F_{2}^{(1)}\left(Y+U^{2} V\right)=$ $F\left(Y+V^{2}+U^{2} V\right)$. On sait que les exposants de la série $\phi-V^{2}$ sont dans le cône affine de sommet $(2,1)$ qui contient les exposants de

$$
\begin{aligned}
F_{3}^{(1)}(0)= & U^{3} V^{6}+2 U^{5} V^{5}+U^{7} V^{4}+U^{4} V^{12}+5 U^{6} V^{11} \\
& +10 U^{8} V^{10}+10 U^{10} V^{9}+5 U^{12} V^{8}+U^{14} V^{7} .
\end{aligned}
$$

C'est-à-dire le cône $(2,1)+\operatorname{pos}\{(1,5),(2,1)\}$.

(Pour $w \in \sigma_{2}$, on définit $F_{2}^{(2)}=F\left(Y+U^{2} V\right)$, on vérifie que $F_{3}^{(2)}=F_{2}^{(2)}\left(Y+V^{2}\right)=$ $F\left(Y+V^{2}+U^{2} V\right)=F_{3}^{(1)}$, et que les exposants de la série construit $\phi-U^{2} V$ sont dans le cône affine $(0,2)+\operatorname{pos}\{(2,5),(7,2)\}$.) 
On obtient que les exposants de $\phi$ sont dans le cône affine $(-2,-2)+\operatorname{pos}\{(2,5),(1,2)\}$. Comme le cône $\Delta^{\vee}=\operatorname{pos}\{(2,5),(1,2)\}$ la série $\phi$ est dans l'anneau $\mathbb{C}\left\{\left\{S_{\Delta}\right\}\right\}$. (Voir la figure 1.)

Pendant la préparation de ce travail, l'auteur a bénéficié du soutien de la DGUI du gouvernement des Iles Canaries, et de l'hospitalité du DMI de l'ENS de Paris.

\section{Références}

[1] S. S. Abhyankar, On the ramification of algebraic functions. Amer. J. Math. 77(1955), 575-592.

[2] M. E. Alonso, I. Luengo et M. Raimondo, An Algorithm on Quasi-Ordinary Polynomials. LNCS (Proc. AAECC-6) 357, Springer-Verlag, 1989, 59-73.

[3] L. J. Billera et B. Sturmfels, Fiber Polytopes. Ann. Math. 135(1992), 527-549.

[4] W. Fulton, Introduction to Toric Varieties. Ann. of Math. Stud. 131, Princeton University Press, 1993.

[5] - Algebraic Topology. Springer-Verlag, 1995.

[6] I. M. Gel'fand, M. M. Kapranov et A. V. Zelevinsky, Discriminants, Resultants and Multi-Dimensional Determinants. Birkhäuser, Boston, 1994.

[7] L. Kaup et B. Kaup, Holomorphic Functions of Several Complex Variables. de Gruyter Stud. Math. 3, 1983.

[8] J. Lipman, Topological invariants of quasi-ordinary singularities. Mem. Amer. Math. Soc. (388) 74(1988), $1-107$.

[9] J. McDonald, Fiber Polytopes and fractional power series. J. Pure Appl. Algebra. 104(1995), 213-233.

[10] T. Oda, Convex Bodies and Algebraic Geometry. Ann. of Math. Stud. 131, Springer-Verlag, 1988.

[11] O. Zariski et P. Samuel, Commutative Algebra, I. Springer-Verlag, 1975.

Université de Paris VII-Denis Diderot

Institut de Mathématiques

2, place Jussieu

75251 Paris cedex 05

France

email: gonzalez@math.jussieu.fr 